\title{
The stable derived category of a noetherian scheme
}

\author{
Henning Krause \\ Dedicated to Claus Michael Ringel on the occasion of his 60th birthday
}

\begin{abstract}
For a noetherian scheme, we introduce its unbounded stable derived category. This leads to a recollement which reflects the passage from the bounded derived category of coherent sheaves to the quotient modulo the subcategory of perfect complexes. Some applications are included, for instance an analogue of maximal Cohen-Macaulay approximations, a construction of Tate cohomology, and an extension of the classical Grothendieck duality. In addition, the relevance of the stable derived category in modular representation theory is indicated.
\end{abstract}

\section{Introduction}

Let $\mathbb{X}$ be a separated noetherian scheme and denote by Qcoh $\mathbb{X}$ the category of quasi-coherent sheaves on $\mathbb{X}$. We consider the derived category $\mathbf{D}($ Qcoh $\mathbb{X})$ and two full subcategories

$$
\mathbf{D}^{\text {perf }}(\operatorname{coh} \mathbb{X}) \subseteq \mathbf{D}^{\mathrm{b}}(\operatorname{coh} \mathbb{X}) \subseteq \mathbf{D}(\mathrm{Q} \operatorname{coh} \mathbb{X})
$$

which are of particular interest. Here, $\mathbf{D}^{\mathrm{b}}(\operatorname{coh} \mathbb{X})$ denotes the bounded derived category of coherent sheaves, and $\mathbf{D}^{\text {perf }}(\operatorname{coh} \mathbb{X})$ denotes the subcategory of perfect complexes.

Now let Inj $\mathbb{X}$ be the full subcategory of injective objects in Qcoh $\mathbb{X}$, and denote by $\mathbf{K}(\operatorname{Inj} \mathbb{X})$ its homotopy category. The composite

$$
Q: \mathbf{K}(\operatorname{Inj} \mathbb{X}) \stackrel{\text { inc }}{\longrightarrow} \mathbf{K}(\mathrm{Q} \operatorname{coh} \mathbb{X}) \stackrel{\text { can }}{\longrightarrow} \mathbf{D}(\mathrm{Q} \operatorname{coh} \mathbb{X})
$$

gives rise to a localization sequence

$$
\mathbf{S}(\mathrm{Q} \operatorname{coh} \mathbb{X}) \stackrel{I}{\longrightarrow} \mathbf{K}(\operatorname{Inj} \mathbb{X}) \stackrel{Q}{\longrightarrow} \mathbf{D}(\mathrm{Q} \operatorname{coh} \mathbb{X})
$$

where $\mathbf{S}($ Qcoh $\mathbb{X})$ denotes the full subcategory of all acyclic complexes in $\mathbf{K}(\operatorname{Inj} \mathbb{X})$. Thus $Q$ induces an equivalence

$$
\mathbf{K}(\operatorname{Inj} \mathbb{X}) / \mathbf{S}(\mathrm{Q} \operatorname{coh} \mathbb{X}) \stackrel{\sim}{\longrightarrow} \mathbf{D}(\mathrm{Q} \operatorname{coh} \mathbb{X}) .
$$

Next we recall that an object $X$ in some category with coproducts is compact if every map $X \rightarrow \coprod_{i} Y_{i}$ into an arbitrary coproduct factors through a finite coproduct. For instance, an object in $\mathbf{D}(\mathrm{Qcoh} \mathbb{X})$ is compact if and only if it is isomorphic to a perfect complex. It is well known that the derived category $\mathbf{D}(\mathrm{Qcoh} \mathbb{X})$ is compactly generated, that is, there is a set of compact objects which generate $\mathbf{D}(\mathrm{Qcoh} \mathbb{X})$ [Nee96]. To formulate our main result, let us denote by $\mathbf{K}^{\mathrm{c}}(\mathrm{Inj} \mathbb{X})$ and $\mathbf{S}^{\mathrm{c}}(\mathrm{Qcoh} \mathbb{X})$ the full subcategories of compact objects in $\mathbf{K}(\operatorname{Inj} \mathbb{X})$ and $\mathbf{S}(\mathrm{Qcoh} \mathbb{X})$, respectively.

Received 22 April 2004, accepted in final form 24 September 2004, published online 1 September 2005. 2000 Mathematics Subject Classification 14F05, 18E30 (primary), 16E45, 16G50, $55 \mathrm{U} 35$ (secondary).

Keywords: stable category, derived category, noetherian scheme, Grothendieck duality, Gorenstein injective, Tate cohomology.

This journal is (C) Foundation Compositio Mathematica 2005. 
Theorem 1.1. Let $\mathbb{X}$ be a separated noetherian scheme.

(1) The functors $I, Q$ have left adjoints $I_{\lambda}, Q_{\lambda}$ and right adjoints $I_{\rho}, Q_{\rho}$ respectively. We have therefore a recollement

$$
\mathbf{S}(\mathrm{Qcoh} \mathbb{X}) \rightleftarrows \mathbf{K}(\operatorname{Inj} \mathbb{X}) \rightleftarrows \mathbf{D}(\mathrm{Q} \operatorname{coh} \mathbb{X})
$$

(2) The triangulated category $\mathbf{K}(\operatorname{Inj} \mathbb{X})$ is compactly generated, and $Q$ induces an equivalence $\mathbf{K}^{\mathrm{c}}(\operatorname{Inj} \mathbb{X}) \rightarrow \mathbf{D}^{\mathrm{b}}(\operatorname{coh} \mathbb{X})$

(3) The sequence

$$
\mathbf{D}(\mathrm{Q} \operatorname{coh} \mathbb{X}) \stackrel{Q_{\lambda}}{\longrightarrow} \mathbf{K}(\operatorname{Inj} \mathbb{X}) \stackrel{I_{\lambda}}{\longrightarrow} \mathbf{S}(\mathrm{Q} \operatorname{coh} \mathbb{X})
$$

is a localization sequence. Therefore $\mathbf{S}(\mathrm{Qcoh} \mathbb{X})$ is compactly generated, and $I_{\lambda} \circ Q_{\rho}$ induces (up to direct factors) an equivalence

$$
\mathbf{D}^{\mathrm{b}}(\operatorname{coh} \mathbb{X}) / \mathbf{D}^{\text {perf }}(\operatorname{coh} \mathbb{X}) \stackrel{\sim}{\longrightarrow} \mathbf{S}^{\mathrm{c}}(\mathrm{Q} \operatorname{coh} \mathbb{X}) .
$$

Note that this theorem is a special case of a general result about Grothendieck categories. All we need is a locally noetherian Grothendieck category $\mathcal{A}$, for instance $\mathcal{A}=$ Qcoh $\mathbb{X}$, such that $\mathbf{D}(\mathcal{A})$ is compactly generated. There is a surprising consequence which seems worth mentioning.

COROLlary 1.2. Let $\mathbb{X}$ be a separated noetherian scheme. Then a product of acyclic complexes of injective objects in Qcoh $\mathbb{X}$ is acyclic.

We call the category $\mathbf{S}($ Qcoh $\mathbb{X})$ the stable derived category of Qcoh $\mathbb{X}$. A first systematic study of the bounded stable derived category

$$
\mathbf{D}^{\mathrm{b}}(\operatorname{coh} \mathbb{X}) / \mathbf{D}^{\text {perf }}(\operatorname{coh} \mathbb{X})
$$

can be found in work of Buchweitz [Buc87]. Unfortunately this beautiful paper has never been published; however see [BEH87]. For a Gorenstein ring $\Lambda$ he identifies the bounded derived category of finitely generated $\Lambda$-modules modulo perfect complexes

$$
\mathbf{D}^{\mathrm{b}}(\bmod \Lambda) / \mathbf{D}^{\text {perf }}(\bmod \Lambda)
$$

with the stable category of maximal Cohen-Macaulay $\Lambda$-modules and with the category of acyclic complexes of finitely generated projective $\Lambda$-modules. The same identification appears in [Ric89] for selfinjective algebras and plays an important role in modular representation theory of finite groups; see also [KV87]. The approach in the present paper differs from that of Buchweitz substantially because we work in the unbounded setting and we use injective objects instead of projectives. This has some advantages. For instance, in any Grothendieck category we always have enough injectives but often not enough projectives. On the other hand, we obtain a recollement in the unbounded setting which does not exist in the bounded setting. In fact, the celebrated theory of maximal CohenMacaulay approximations [AB89] is described as 'decomposition' [AB89] or 'glueing' [Buc87], but finds a natural interpretation as 'recollement' in the sense of [BBD82] if one passes to the unbounded setting. To be precise, the recollement

$$
\mathbf{S}(\operatorname{Mod} \Lambda) \underset{I_{\lambda}}{\stackrel{I_{\rho}}{\leftrightarrows}} \mathbf{K}(\operatorname{Inj} \Lambda) \underset{Q_{\lambda}}{\stackrel{Q_{\rho}}{\leftrightarrows}} \mathbf{D}(\operatorname{Mod} \Lambda)
$$

induces for any Gorenstein ring $\Lambda$ the Gorenstein injective approximation functor

$$
T: \operatorname{Mod} \Lambda \stackrel{\text { can }}{\longrightarrow} \mathbf{D}(\operatorname{Mod} \Lambda) \stackrel{I_{\lambda} \circ Q_{\rho}}{\longrightarrow} \mathbf{S}(\operatorname{Mod} \Lambda) \stackrel{Z^{0}}{\longrightarrow} \underline{\operatorname{Mod}} \Lambda
$$

where $\underline{\operatorname{Mod}} \Lambda$ denotes the stable category modulo injective objects. For any $\Lambda$-module $A$, the Gorenstein injective approximation $A \rightarrow T A$ is the 'dual' of the maximal Cohen-Macaulay 


\section{H. KRAUSE}

approximation which is based on projective resolutions. Let us stress again that this approach generalizes to any locally noetherian Grothendieck category $\mathcal{A}$ provided that $\mathbf{D}(\mathcal{A})$ is compactly generated.

Next we explain the connection between Gorenstein injective approximations and Tate cohomology. We fix a locally noetherian Grothendieck category $\mathcal{A}$ and pass from the stable derived category $\mathbf{S}(\mathcal{A})$ to the full subcategory $\mathbf{T}(\mathcal{A})$ of totally acyclic complexes. An object in $\mathcal{A}$ is by definition Gorenstein injective if it is of the form $\operatorname{Ker}\left(X^{0} \rightarrow X^{1}\right)$ for some $X$ in $\mathbf{T}(\mathcal{A})$. The inclusion $G: \mathbf{T}(\mathcal{A}) \rightarrow \mathbf{K}(\operatorname{Inj} \mathcal{A})$ has a left adjoint $G_{\lambda}$. Given an object $A$ in $\mathcal{A}$ with injective resolution $i A$, we may think of $G_{\lambda} i A$ as a complete injective resolution of $A$. This leads to the following definition of Tate cohomology groups

$$
\widehat{\operatorname{Ext}}_{\mathcal{A}}^{n}(A, B)=H^{n} \operatorname{Hom}_{\mathcal{A}}\left(A, G_{\lambda} i B\right)
$$

for any $A, B$ in $\mathcal{A}$ and $n \in \mathbb{Z}$. This cohomology theory is symmetric in the sense that for any $A$ in $\mathcal{A}$, we have

$$
\widehat{\operatorname{Ext}}_{\mathcal{A}}^{*}(A,-)=0 \Longleftrightarrow \widehat{\operatorname{Ext}}_{\mathcal{A}}^{*}(-, A)=0 \Longleftrightarrow \widehat{\operatorname{Ext}}_{\mathcal{A}}^{0}(A, A)=0
$$

Let $\mathcal{X}$ denote the class of all objects $A$ such that $\widehat{\operatorname{Ext}}_{\mathcal{A}}^{*}(A,-)$ vanishes, and let $\mathcal{Y}$ be the class of Gorenstein injective objects in $\mathcal{A}$.

Theorem 1.3. Let $\mathcal{A}$ be a locally noetherian Grothendieck category and suppose that $\mathbf{D}(\mathcal{A})$ is compactly generated.

(1) $\mathcal{X}=\left\{A \in \mathcal{A} \mid \operatorname{Ext}_{\mathcal{A}}^{1}(A, B)=0\right.$ for all $\left.B \in \mathcal{Y}\right\}$.

(2) $\mathcal{Y}=\left\{B \in \mathcal{A} \mid \operatorname{Ext}_{\mathcal{A}}^{1}(A, B)=0\right.$ for all $\left.A \in \mathcal{X}\right\}$.

(3) Every object $A$ in $\mathcal{A}$ fits into exact sequences

$$
0 \rightarrow Y_{A} \rightarrow X_{A} \rightarrow A \rightarrow 0 \quad \text { and } \quad 0 \rightarrow A \rightarrow Y^{A} \rightarrow X^{A} \rightarrow 0
$$

in $\mathcal{A}$ with $X_{A}, X^{A}$ in $\mathcal{X}$ and $Y_{A}, Y^{A}$ in $\mathcal{Y}$.

(4) $\mathcal{X} \cap \mathcal{Y}=\operatorname{Inj} \mathcal{A}$.

After explaining some historical background, let us mention more recent work on stable derived categories. For instance, Beligiannis developed a general theory of 'stabilization' in the framework of relative homological algebra [Bel00], and Jørgensen studied the category of 'spectra' for a module category [Jor01]. Also, Orlov discussed the category

$$
\mathbf{D}^{\mathrm{b}}(\operatorname{coh} \mathbb{X}) / \mathbf{D}^{\text {perf }}(\operatorname{coh} \mathbb{X})
$$

under the name 'triangulated category of singularities' and pointed out some connection with the Homological Mirror Symmetry Conjecture [Orl03]. In any case, our notation $\mathbf{S}($ Qcoh $\mathbb{X})$ reflects this terminology.

Our main results suggests that the homotopy category $\mathbf{K}(\operatorname{Inj} \mathbb{X})$ deserves some more attention. We may think of this category as the 'compactly generated completion' of the category $\mathbf{D}^{\mathrm{b}}(\operatorname{coh} \mathbb{X})$. In fact, the category coh $\mathbb{X}$ of coherent sheaves carries a natural differential graded (DG) structure and its derived category $\mathbf{D}_{\mathrm{dg}}(\operatorname{coh} \mathbb{X})$ is equivalent to $\mathbf{K}(\operatorname{Inj} \mathbb{X})$. This follows from Keller's work [Kel94] and complements a recent result of Bondal and van den Bergh [BV03] which says that $\mathbf{D}(\mathrm{Q} \operatorname{coh} \mathbb{X})$ is equivalent to $\mathbf{D}_{\mathrm{dg}}(A)$ for some DG algebra $A$.

As another application of our main result, let us mention that the adjoint pair of functors $\mathbf{R} f_{*}$ and $f^{!}$which establish the Grothendieck duality for a morphism $f: \mathbb{X} \rightarrow \mathbb{Y}$ between schemes [Har66, Nee96], can be extended to a pair of adjoint functors between $\mathbf{K}(\operatorname{Inj} \mathbb{X})$ and $\mathbf{K}(\operatorname{Inj} \mathbb{Y})$. 
Theorem 1.4. Let $f: \mathbb{X} \rightarrow \mathbb{Y}$ be a morphism between separated noetherian schemes. Denote by $\mathbf{R} f_{*}: \mathbf{D}(\mathrm{Qcoh} \mathbb{X}) \rightarrow \mathbf{D}($ Qcoh $\mathbb{Y})$ the right derived direct image functor and by $f^{!}$its right adjoint. Then there is an adjoint pair of functors $\hat{\mathbf{R}} f_{*}$ and $\widehat{f} !$ making the following diagrams commutative.
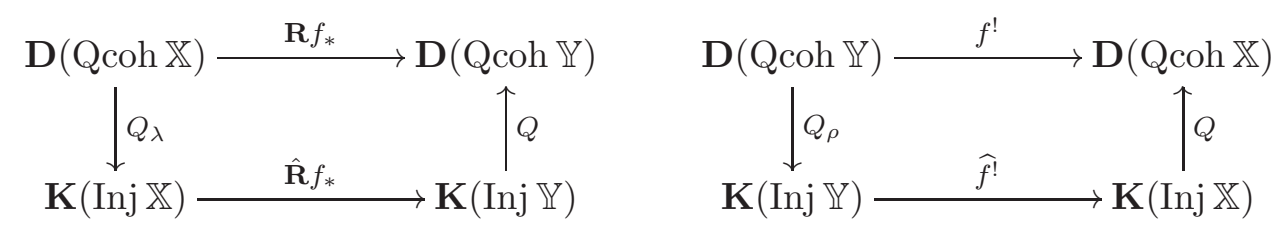

Again, this theorem is really a lot more general. It is irrelevant that the functor $f_{*}$ comes from a morphism $f: \mathbb{X} \rightarrow \mathbb{Y}$. All we need is that $f_{*}$ and its right derived functor $\mathbf{R} f_{*}$ preserve coproducts. On the other hand, there is a strengthened version of Theorem 1.4 which uses the special properties of $f_{*}$. The author is grateful to Amnon Neeman for pointing out the following.

Theorem 1.5 (Neeman). Let $f: \mathbb{X} \rightarrow \mathbb{Y}$ be a morphism between separated noetherian schemes. Then $\hat{\mathbf{R}} f_{*}$ sends acyclic complexes to acyclic complexes. Thus we have an adjoint pair of functors between $\mathbf{S}($ Qcoh $\mathbb{X})$ and $\mathbf{S}($ Qcoh $\mathbb{Y})$, making the following diagrams commutative.

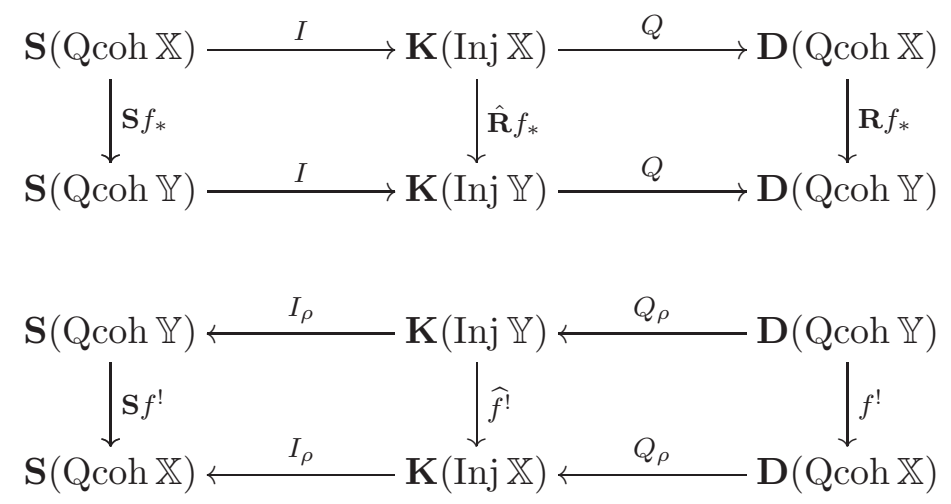

It seems an interesting project to study the functor $\mathbf{S} f_{*}$, for instance to find out when it is an equivalence. The following result demonstrates the geometric content of this question; it generalizes a result of Orlov for the bounded stable derived category [Orl03].

Theorem 1.6. Let $\mathbb{Y}$ be a separated noetherian scheme of finite Krull dimension. If $f: \mathbb{X} \rightarrow \mathbb{Y}$ denotes the inclusion of an open subscheme which contains all singular points of $\mathbb{Y}$, then $\mathbf{S} f_{*}: \mathbf{S}(\mathrm{Qcoh} \mathbb{X}) \rightarrow \mathbf{S}($ Qcoh $\mathbb{Y})$ is an equivalence.

Despite the title of this paper and the algebraic geometric formulation of the main results, there is another source of serious interest in stable categories. Take a finite group $G$ and a field $k$. A classical object in modular representation theory is the stable module category $\underline{\operatorname{Mod}} k G$ of the group algebra $k G$. We shall see that this stable category is equivalent to the stable derived category of the full module category $\operatorname{Mod} k G$. Using a slightly different setting, Hovey et al. studied the functor

$$
I_{\lambda}: \mathbf{K}(\operatorname{Inj} k G) \longrightarrow \mathbf{S}(\operatorname{Mod} k G) \cong \underline{\operatorname{Mod}} k G
$$

in their work on axiomatic stable homotopy theory [HPS97]. Note that $\mathbf{K}(\operatorname{Inj} k G)$ carries a commutative tensor product and the (graded) endomorphism ring of its unit is simply the group cohomology ring $H^{*}(G, k)$. Therefore, $\mathbf{K}(\operatorname{Inj} k G)$ seems to be the right object for studying representations of $G$ via methods from commutative algebra. In fact, the composite

$$
\mathbf{D}(\operatorname{Mod} k G) \stackrel{I_{\lambda} \circ Q_{\rho}}{\longrightarrow} \mathbf{S}(\operatorname{Mod} k G) \stackrel{Z^{0}}{\longrightarrow} \underline{\operatorname{Mod}} k G
$$

plays a crucial role in recent work of Benson and Greenlees [BG04]. 


\section{H. KRAUSE}

Having stated some of the main results, let us sketch the outline of this paper. The paper deals with locally noetherian Grothendieck categories and covers therefore various applications, for instance in algebraic geometry or representation theory. Thus we fix a locally noetherian Grothendieck category $\mathcal{A}$ and study the recollement

$$
\mathbf{S}(\mathcal{A}) \rightleftarrows \mathbf{K}(\operatorname{Inj} \mathcal{A}) \rightleftarrows \mathbf{D}(\mathcal{A})
$$

More specifically, we begin in $\S 2$ with the basic properties of the homotopy category $\mathbf{K}(\operatorname{Inj} \mathcal{A})$. The recollement (1.1) is established in $\S \S 3$ and 4 . In $\S 5$, we discuss the essential properties of the stable derived category $\mathbf{S}(\mathcal{A})$. Then we extend derived functors in $\S 6$, and $\S 7$ is devoted to studying Gorenstein injective approximations and Tate cohomology. In $\S 8$, we indicate the relevance of the stable derived category in modular representation theory. Appendix A provides additional material about DG categories, and Appendix B discusses homotopically minimal complexes.

\section{The homotopy category of injectives}

We fix a locally noetherian Grothendieck category $\mathcal{A}$. Thus, $\mathcal{A}$ is an abelian Grothendieck category and has a set $\mathcal{A}_{0}$ of noetherian objects which generate $\mathcal{A}$; that is, every object in $\mathcal{A}$ is a quotient of a coproduct of objects in $\mathcal{A}_{0}$. We denote by noeth $\mathcal{A}$ the full subcategory formed by the noetherian objects in $\mathcal{A}$, and $\operatorname{Inj} \mathcal{A}$ denotes the full subcategory of injective objects. Note that $\operatorname{Inj} \mathcal{A}$ is closed under taking coproducts.

We write $\mathbf{K}(\mathcal{A})$ for the homotopy category and $\mathbf{D}(\mathcal{A})$ for the derived category of unbounded complexes in $\mathcal{A}$; for their definitions and basic properties, we refer to [Ver96]. We do not distinguish between an object in $\mathcal{A}$ and the corresponding complex concentrated in degree zero in the homotopy category $\mathbf{K}(\mathcal{A})$. The inclusion noeth $\mathcal{A} \rightarrow \mathcal{A}$ induces a fully faithful functor

$$
\mathbf{D}^{\mathrm{b}}(\operatorname{noeth} \mathcal{A}) \longrightarrow \mathbf{D}(\mathcal{A})
$$

which identifies $\mathbf{D}^{\mathrm{b}}($ noeth $\mathcal{A})$ with the full subcategory of objects $X$ in $\mathbf{D}(\mathcal{A})$ such that $H^{n} X$ is noetherian for all $n$ and $H^{n} X=0$ for almost all $n \in \mathbb{Z}$; see [Ver96, Proposition III.2.4.1].

In this section, we study the basic properties of the homotopy category $\mathbf{K}(\operatorname{Inj} \mathcal{A})$. We shall see that this category solves a completion problem for the triangulated category $\mathbf{D}^{\mathrm{b}}$ (noeth $\mathcal{A}$ ). Let us begin with some elementary observations.

Lemma 2.1. Let $A$ be an object in $\mathcal{A}$ and denote by $i A$ an injective resolution. Then the natural map

$$
\operatorname{Hom}_{\mathbf{K}(\mathcal{A})}(i A, X) \longrightarrow \operatorname{Hom}_{\mathbf{K}(\mathcal{A})}(A, X)
$$

is an isomorphism for all $X$ in $\mathbf{K}(\operatorname{Inj} \mathcal{A})$. Therefore, $i A$ is a compact object in $\mathbf{K}(\operatorname{Inj} \mathcal{A})$ if $A$ is noetherian.

Proof. Denote for any $n \in \mathbb{Z}$ by $\sigma^{\geqslant n} X$ the truncation satisfying

$$
\left(\sigma^{\geqslant n} X\right)^{p}= \begin{cases}X^{p} & \text { if } p \geqslant n, \\ 0 & \text { if } p<n .\end{cases}
$$

We complete the map $A \rightarrow i A$ to an exact triangle

$$
a A \longrightarrow A \longrightarrow i A \longrightarrow \Sigma(a A)
$$

and obtain

$$
\operatorname{Hom}_{\mathbf{K}(\mathcal{A})}(a A, X) \cong \operatorname{Hom}_{\mathbf{K}(\mathcal{A})}\left(a A, \sigma^{\geqslant-1} X\right)=0
$$

since $a A$ is acyclic and concentrated in non-negative degrees. Thus,

$$
\operatorname{Hom}_{\mathbf{K}(\mathcal{A})}(i A, X) \cong \operatorname{Hom}_{\mathbf{K}(\mathcal{A})}(A, X) .
$$


Now assume that $A$ is noetherian. Clearly, $A$ is a compact object in $\mathcal{A}$ and therefore a compact object in $\mathbf{K}(\mathcal{A})$. The isomorphism $(2.1)$ shows that $i A$ is a compact object in $\mathbf{K}(\operatorname{Inj} \mathcal{A})$.

Lemma 2.2. Let $X$ be a non-zero object in $\mathbf{K}(\operatorname{Inj} \mathcal{A})$. Then there exists a noetherian object $A$ in $\mathcal{A}$ such that $\operatorname{Hom}_{\mathbf{K}(\mathcal{A})}\left(A, \Sigma^{n} X\right) \neq 0$ for some $n \in \mathbb{Z}$.

Proof. Suppose first that $H^{n} X \neq 0$ for some $n$. Choose a noetherian object $A$ and a map $A \rightarrow Z^{n} X$ inducing a non-zero map $A \rightarrow H^{n} X$. We obtain a chain map $A \rightarrow \Sigma^{n} X$ which induces a non-zero element in $\operatorname{Hom}_{\mathbf{K}(\mathcal{A})}\left(A, \Sigma^{n} X\right)$.

Now suppose $H^{n} X=0$ for all $n$. We can choose $n$ such that $Z^{n} X$ is non-injective. Using Baer's criterion, there exists a noetherian object $A$ in $\mathcal{A}$ such that $\operatorname{Ext}_{\mathcal{A}}^{1}\left(A, Z^{n} X\right)$ is non-zero. Now observe that

$$
\operatorname{Hom}_{\mathbf{K}(\mathcal{A})}\left(A, \Sigma^{n+p} X\right) \cong \operatorname{Ext}_{\mathcal{A}}^{p}\left(A, Z^{n} X\right)
$$

for all $p \geqslant 1$. Thus $\operatorname{Hom}_{\mathbf{K}(\mathcal{A})}\left(A, \Sigma^{n+1} X\right) \neq 0$. This completes the proof.

Let $\mathcal{T}$ be a triangulated category with arbitrary coproducts. Recall that an object $X$ in $\mathcal{T}$ is compact if $\operatorname{Hom}_{\mathcal{T}}(X,-)$ preserves all coproducts. The triangulated category is compactly generated if there is a set $\mathcal{T}_{0}$ of compact objects such that $\operatorname{Hom}_{\mathcal{T}}\left(X, \Sigma^{n} Y\right)=0$ for all $X \in \mathcal{T}_{0}$ and $n \in \mathbb{Z}$ implies $Y=0$ for every object $Y$ in $\mathcal{T}$.

Proposition 2.3. Let $\mathcal{A}$ be a locally noetherian Grothendieck category, and let $\mathbf{K}^{\mathrm{c}}(\operatorname{Inj} \mathcal{A})$ denote the full subcategory of compact objects in $\mathbf{K}(\operatorname{Inj} \mathcal{A})$.

(1) The triangulated category $\mathbf{K}(\operatorname{Inj} \mathcal{A})$ is compactly generated.

(2) The canonical functor $\mathbf{K}(\mathcal{A}) \rightarrow \mathbf{D}(\mathcal{A})$ induces an equivalence

$$
\mathbf{K}^{\mathrm{c}}(\operatorname{Inj} \mathcal{A}) \stackrel{\sim}{\longrightarrow} \mathbf{D}^{\mathrm{b}}(\text { noeth } \mathcal{A}) .
$$

Proof. It follows from Lemmas 2.1 and 2.2 that $\mathbf{K}(\operatorname{Inj} \mathcal{A})$ is compactly generated. A standard argument shows that $\mathbf{K}^{\mathrm{c}}(\operatorname{Inj} \mathcal{A})$ equals the thick subcategory of $\mathbf{K}(\operatorname{Inj} \mathcal{A})$ which is generated by the injective resolutions of the noetherian objects in $\mathcal{A}$; see [Nee92, Lemma 2.2]. The equivalence $\mathbf{K}^{+}(\operatorname{Inj} \mathcal{A}) \rightarrow \mathbf{D}^{+}(\mathcal{A})$ restricts to an equivalence $\mathbf{K}^{+, \mathrm{b}}(\operatorname{Inj} \mathcal{A}) \rightarrow \mathbf{D}^{\mathrm{b}}(\mathcal{A})$ and identifies $\mathbf{K}^{\mathrm{c}}(\operatorname{Inj} \mathcal{A})$ with $\mathbf{D}^{\mathrm{b}}($ noeth $\mathcal{A})$.

Note that we obtain a functor $\mathbf{D}^{\mathrm{b}}(\operatorname{noeth} \mathcal{A}) \rightarrow \mathbf{K}(\operatorname{Inj} \mathcal{A})$ which identifies $\mathbf{D}^{\mathrm{b}}($ noeth $\mathcal{A})$ with the full subcategory of compact objects. Therefore, the formation of the category $\mathbf{K}(\operatorname{Inj} \mathcal{A})$ solves a completion problem which we explain by an analogy. The category $\mathcal{A}$ is a completion of noeth $\mathcal{A}$ in the following sense.

- $\mathcal{A}$ is an additive category with filtered colimits.

- The inclusion noeth $\mathcal{A} \rightarrow \mathcal{A}$ identifies noeth $\mathcal{A}$ with the full subcategory of finitely presented objects.

- $\mathcal{A}$ coincides with the smallest subcategory which contains all finitely presented objects and is closed under forming filtered colimits.

Recall that an object $X$ in $\mathcal{A}$ is finitely presented if the functor $\operatorname{Hom}_{\mathcal{A}}(X,-)$ preserves filtered colimits. Similarly, we have the following for $\mathcal{T}=\mathbf{K}(\operatorname{Inj} \mathcal{A})$.

- $\mathcal{T}$ is a triangulated category with coproducts.

- The functor $\mathbf{D}^{\mathrm{b}}($ noeth $\mathcal{A}) \rightarrow \mathcal{T}$ identifies $\mathbf{D}^{\mathrm{b}}$ (noeth $\mathcal{A}$ ) with the full subcategory of compact objects.

- $\mathcal{T}$ coincides with the smallest subcategory which contains all compact objects and is closed under forming triangles and coproducts. 


\section{H. KRAUSE}

The category $\mathcal{A}$ is, up to an equivalence, uniquely determined by noeth $\mathcal{A}$. It would be interesting to know to what extent $\mathbf{K}(\operatorname{Inj} \mathcal{A})$ is uniquely determined by $\mathbf{D}^{\mathrm{b}}(\operatorname{noeth} \mathcal{A})$.

Example 2.4. Suppose there is a noetherian object $A$ in $\mathcal{A}$ such that $\mathbf{D}^{\mathrm{b}}($ noeth $\mathcal{A}$ ) is generated by $A$, that is, there is no proper thick subcategory containing $A$. Take an injective resolution $i A$ and denote by $\mathcal{E} n d_{\mathcal{A}}(A)$ the endomorphism DG algebra of $i A$. Then $\mathcal{H o m}_{\mathcal{A}}(i A,-)$ induces an equivalence between $\mathbf{K}(\operatorname{Inj} \mathcal{A})$ and the derived category $\mathbf{D}_{\mathrm{dg}}\left(\mathcal{E} n d_{\mathcal{A}}(A)\right)$ of $\mathrm{DG} \mathcal{E} n d_{\mathcal{A}}(A)$-modules; see [Kel94]. If one replaces a single generator by a set of generating objects, then one obtains an analogue which involves a DG category instead of a DG algebra. In particular, noeth $\mathcal{A}$ carries the structure of a $D G$ category such that $\mathbf{K}(\operatorname{Inj} \mathcal{A})$ and $\mathbf{D}_{\mathrm{dg}}($ noeth $\mathcal{A})$ are equivalent. We refer to Appendix A for details.

Example 2.5. Let $G$ be a finite $p$-group and $k$ be a field of characteristic $p>0$. We consider the category $\mathcal{A}=\operatorname{Mod} k G$ of modules over the group algebra $k G$. Take an injective resolution $i k$ of the trivial representation $k$, and denote by $\mathcal{E}_{n} d_{k G}(k)$ the endomorphism DG algebra of $i k$. Then its derived category $\mathbf{D}_{\mathrm{dg}}\left(\mathcal{E} n d_{k G}(k)\right)$ is equivalent to $\mathbf{K}(\operatorname{Inj} \mathcal{A})$. The tensor product $\otimes_{k}$ on $\mathcal{A}$ restricts to a product on $\operatorname{Inj} \mathcal{A}$ and therefore induces a (total) tensor product on $\mathbf{K}(\operatorname{Inj} \mathcal{A})$. On the other hand, the $E_{\infty}$-structure of $\mathcal{E} n d_{k G}(k)$ induces a product on $\mathbf{D}_{\mathrm{dg}}\left(\mathcal{E} n d_{k G}(k)\right)$. We conjecture that these products are naturally isomorphic.

Example 2.6. Let $\Lambda$ be a finite-dimensional algebra over a field $k$. Then $E=\operatorname{Hom}_{k}\left(\Lambda^{\mathrm{op}}, k\right)$ is an injective cogenerator for $\mathcal{A}=\operatorname{Mod} \Lambda$, and $\operatorname{Hom}_{\Lambda}(E,-)$ induces an equivalence $\operatorname{Inj} \mathcal{A} \rightarrow$ Proj $\mathcal{A}$ since $\operatorname{Hom}_{\Lambda}(E, E) \cong \Lambda$. Thus, the homotopy category $\mathbf{K}(\operatorname{Proj} \mathcal{A})$ is compactly generated. For more on $\mathbf{K}(\operatorname{Proj} \mathcal{A})$, see [Jor01, Jor05].

\section{A localization sequence}

Let $\mathcal{A}$ be a locally noetherian Grothendieck category and let

$$
\mathbf{K}_{\mathrm{ac}}(\operatorname{Inj} \mathcal{A})=\mathbf{K}(\operatorname{Inj} \mathcal{A}) \cap \mathbf{K}_{\mathrm{ac}}(\mathcal{A}),
$$

where $\mathbf{K}_{\mathrm{ac}}(\mathcal{A})$ denotes the full subcategory formed by all acyclic complexes in $\mathbf{K}(\mathcal{A})$. In this section, we prove that the canonical functors

$$
I: \mathbf{K}_{\mathrm{ac}}(\operatorname{Inj} \mathcal{A}) \stackrel{\mathrm{inc}}{\longrightarrow} \mathbf{K}(\operatorname{Inj} \mathcal{A}) \quad \text { and } \quad Q: \mathbf{K}(\operatorname{Inj} \mathcal{A}) \stackrel{\mathrm{inc}}{\longrightarrow} \mathbf{K}(\mathcal{A}) \stackrel{\text { can }}{\longrightarrow} \mathbf{D}(\mathcal{A})
$$

form a localization sequence

$$
\mathbf{K}_{\mathrm{ac}}(\operatorname{Inj} \mathcal{A}) \stackrel{I}{\longrightarrow} \mathbf{K}(\operatorname{Inj} \mathcal{A}) \stackrel{Q}{\longrightarrow} \mathbf{D}(\mathcal{A}) .
$$

Let us start with some preparations. In particular, we need to give the definition of a localization sequence.

DeFinition 3.1. We say that a sequence

$$
\mathcal{T}^{\prime} \stackrel{F}{\longrightarrow} \mathcal{T} \stackrel{G}{\longrightarrow} \mathcal{T}^{\prime \prime}
$$

of exact functors between triangulated categories is a localization sequence if the following holds.

(L1) The functor $F$ has a right adjoint $F_{\rho}: \mathcal{T} \rightarrow \mathcal{T}^{\prime}$ satisfying $F_{\rho} \circ F \cong \operatorname{Id}_{\mathcal{T}^{\prime}}$.

(L2) The functor $G$ has a right adjoint $G_{\rho}: \mathcal{T}^{\prime \prime} \rightarrow \mathcal{T}$ satisfying $G \circ G_{\rho} \cong \operatorname{Id}_{\mathcal{T}^{\prime \prime}}$.

(L3) Let $X$ be an object in $\mathcal{T}$. Then $G X=0$ if and only if $X \cong F X^{\prime}$ for some $X^{\prime} \in \mathcal{T}^{\prime}$.

The sequence $(F, G)$ of functors is called a colocalization sequence if the sequence $\left(F^{\mathrm{op}}, G^{\mathrm{op}}\right)$ of opposite functors is a localization sequence.

The basic properties of a localization sequence are the following [Ver96, § II.2]. 
(1) The functors $F$ and $G_{\rho}$ are fully faithful.

(2) Identify $\mathcal{T}^{\prime}=\operatorname{Im} F$ and $\mathcal{T}^{\prime \prime}=\operatorname{Im} G_{\rho}$. Given objects $X, Y \in \mathcal{T}$, then

$$
\begin{aligned}
X \in \mathcal{T}^{\prime} & \Longleftrightarrow \operatorname{Hom}_{\mathcal{T}}\left(X, \mathcal{T}^{\prime \prime}\right)=0, \\
Y \in \mathcal{T}^{\prime \prime} & \Longleftrightarrow \operatorname{Hom}_{\mathcal{T}}\left(\mathcal{T}^{\prime}, Y\right)=0 .
\end{aligned}
$$

(3) Identify $\mathcal{T}^{\prime}=\operatorname{Im} F$. Then the functor $G$ induces an equivalence $\mathcal{T} / \mathcal{T}^{\prime} \rightarrow \mathcal{T}^{\prime \prime}$.

(4) Let $X$ be an object in $\mathcal{T}$. Then there is an exact triangle

$$
\left(F \circ F_{\rho}\right) X \longrightarrow X \longrightarrow\left(G_{\rho} \circ G\right) X \longrightarrow \Sigma\left(\left(F \circ F_{\rho}\right) X\right)
$$

which is functorial in $X$.

(5) The sequence

$$
\mathcal{T}^{\prime \prime} \stackrel{G_{\rho}}{\longrightarrow} \mathcal{T} \stackrel{F_{\rho}}{\longrightarrow} \mathcal{T}^{\prime}
$$

is a colocalization sequence.

The next lemma is well known; it provides useful criteria for a sequence to be a localization sequence. Recall that a full subcategory of a triangulated category is thick if it is a triangulated subcategory which is closed under taking direct factors.

Lemma 3.2. Let $\mathcal{T}$ be a triangulated category and $\mathcal{S}$ be a thick subcategory. Then the following are equivalent.

(1) The sequence $\mathcal{S} \stackrel{\text { inc }}{\longrightarrow} \mathcal{T} \stackrel{\text { can }}{\longrightarrow} \mathcal{T} / \mathcal{S}$ is a localization sequence.

(2) The inclusion functor $\mathcal{S} \rightarrow \mathcal{T}$ has a right adjoint.

(3) The quotient functor $\mathcal{T} \rightarrow \mathcal{T} / \mathcal{S}$ has a right adjoint.

Proof. Condition (1) implies (2) and (3). Also, (2) and (3) together imply (1). Thus we need to show that (2) and (3) are equivalent. Let us write $F: \mathcal{S} \rightarrow \mathcal{T}$ and $G: \mathcal{T} \rightarrow \mathcal{T} / \mathcal{S}$ for the functors which are involved.

$(2) \Rightarrow(3)$ We obtain a functor $L: \mathcal{T} \rightarrow \mathcal{T}$ by completing for each $X$ in $\mathcal{T}$ the natural map $\left(F \circ F_{\rho}\right) X \rightarrow X$ to an exact triangle

$$
\left(F \circ F_{\rho}\right) X \longrightarrow X \longrightarrow L X \longrightarrow \Sigma\left(\left(F \circ F_{\rho}\right) X\right) .
$$

The functor $L$ annihilates $\mathcal{S}$ and therefore factors through $G$ via an exact functor $G_{\rho}: \mathcal{T} / \mathcal{S} \rightarrow \mathcal{T}$. This is a right adjoint of $G$. In fact, for each pair of objects $X$ in $\mathcal{T}$ and $Y$ in $\mathcal{T} / \mathcal{S}$, the natural map

$$
\operatorname{Hom}_{\mathcal{T} / \mathcal{S}}(G X, Y) \longrightarrow \operatorname{Hom}_{\mathcal{T}}\left(L X, G_{\rho} Y\right) \longrightarrow \operatorname{Hom}_{\mathcal{T}}\left(X, G_{\rho} Y\right)
$$

is bijective.

$(3) \Rightarrow(2)$ We obtain a right adjoint $F_{\rho}: \mathcal{T} \rightarrow \mathcal{S}$ for the inclusion $F$ by completing for each $X$ in $\mathcal{T}$ the natural map $X \rightarrow\left(G_{\rho} \circ G\right) X$ to an exact triangle

$$
F_{\rho} X \longrightarrow X \longrightarrow\left(G_{\rho} \circ G\right) X \longrightarrow \Sigma\left(F_{\rho} X\right) .
$$

Note that $F_{\rho} X$ belongs to $\mathcal{S}$ since $G\left(F_{\rho} X\right)=0$.

We need to construct left and right adjoints for functors starting in a compactly generated triangulated category. Our basic tool for this is the following result which is due to Neeman.

Proposition 3.3. Let $F: \mathcal{S} \rightarrow \mathcal{T}$ be an exact functor between triangulated categories, and suppose $\mathcal{S}$ is compactly generated.

(1) There is a right adjoint $\mathcal{T} \rightarrow \mathcal{S}$ if and only if $F$ preserves all coproducts.

(2) There is a left adjoint $\mathcal{T} \rightarrow \mathcal{S}$ if and only if $F$ preserves all products. 


\section{H. KRAUSE}

Proof. For (1), see [Nee96, Theorem 4.1]. The proof of (2) is analogous and uses covariant Brown representability [Nee01, Theorem 8.6.1]; see also [Kra02].

We record a similar result for later use.

Proposition 3.4. Let $\mathcal{T}$ be a compactly generated triangulated category and $\mathcal{S}_{0}$ be a set of objects in $\mathcal{T}$. Denote by $\mathcal{U}$ the full subcategory of objects $Y$ in $\mathcal{T}$ such that $\operatorname{Hom}_{\mathcal{T}}\left(\Sigma^{n} X, Y\right)=0$ for all $X \in \mathcal{S}_{0}$ and $n \in \mathbb{Z}$. Then the inclusion $\mathcal{U} \rightarrow \mathcal{T}$ has a left adjoint.

Proof. The localizing subcategory $\mathcal{S}$ generated by $\mathcal{S}_{0}$ is well generated and the inclusion $\mathcal{S} \rightarrow \mathcal{T}$ therefore has a right adjoint; see [Nee01]. We obtain a localization sequence $\mathcal{S} \stackrel{\text { inc }}{\longrightarrow} \mathcal{T} \stackrel{\text { can }}{\longrightarrow} \mathcal{T} / \mathcal{S}$ by Lemma 3.2, and the right adjoint of the canonical functor $\mathcal{T} \rightarrow \mathcal{T} / \mathcal{S}$ identifies $\mathcal{T} / \mathcal{S}$ with $\mathcal{U}$.

There is a useful criterion when a left adjoint preserves compactness.

Lemma 3.5. Let $F: \mathcal{S} \rightarrow \mathcal{T}$ be an exact functor between compactly generated triangulated categories which has a right adjoint $G$. Then $F$ preserves compactness if and only if $G$ preserves coproducts.

Proof. See [Nee96, Theorem 5.1].

The following result establishes the localization sequence for the homotopy category of injective objects.

Proposition 3.6. Let $\mathcal{A}$ be a locally noetherian Grothendieck category. Then the canonical functors $\mathbf{K}_{\mathrm{ac}}(\operatorname{Inj} \mathcal{A}) \rightarrow \mathbf{K}(\operatorname{Inj} \mathcal{A})$ and $\mathbf{K}(\operatorname{Inj} \mathcal{A}) \rightarrow \mathbf{D}(\mathcal{A})$ form a localization sequence

$$
\mathbf{K}_{\mathrm{ac}}(\operatorname{Inj} \mathcal{A}) \stackrel{I}{\longrightarrow} \mathbf{K}(\operatorname{Inj} \mathcal{A}) \stackrel{Q}{\longrightarrow} \mathbf{D}(\mathcal{A}) .
$$

Proof. We know from Proposition 2.3 that $\mathbf{K}(\operatorname{Inj} \mathcal{A})$ is compactly generated. In addition, we use Lemma 3.2 and Proposition 3.3. The inclusion $J: \mathbf{K}(\operatorname{Inj} \mathcal{A}) \rightarrow \mathbf{K}(\mathcal{A})$ preserves products and therefore has a left adjoint $J_{\lambda}$ satisfying $J_{\lambda} \circ J \cong \operatorname{Id}_{\mathbf{K}(\operatorname{Inj} \mathcal{A})}$. We obtain a localization sequence

$$
\mathcal{K} \stackrel{\text { inc }}{\longrightarrow} \mathbf{K}(\mathcal{A}) \stackrel{J_{\lambda}}{\longrightarrow} \mathbf{K}(\operatorname{Inj} \mathcal{A})
$$

where $\mathcal{K}$ denotes the kernel of $J_{\lambda}$. Thus

$$
\operatorname{Hom}_{\mathbf{K}(\mathcal{A})}(X, Y)=0 \quad \text { for all } X \in \mathcal{K} \text { and } Y \in \mathbf{K}(\operatorname{Inj} \mathcal{A}) \text {. }
$$

This implies that $\mathcal{K} \subseteq \mathbf{K}_{\mathrm{ac}}(\mathcal{A})$ and gives the following commutative diagram of exact functors.

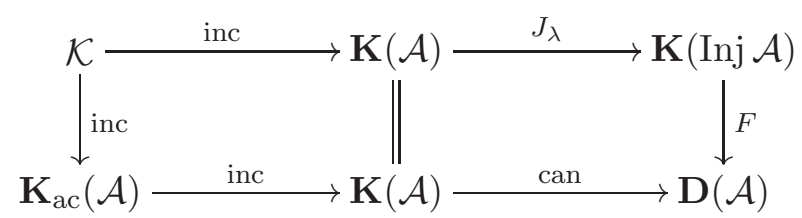

The functor $F$ is induced by the canonical functor $\mathbf{K}(\mathcal{A}) \rightarrow \mathbf{D}(\mathcal{A})$, and we have $F \cong Q$ since $J_{\lambda} \circ J \cong \operatorname{Id}_{\mathbf{K}(\operatorname{Inj} \mathcal{A})}$. Moreover, $F$ preserves coproducts and has therefore a right adjoint $F_{\rho}$. The composite $J \circ F_{\rho}$ is a right adjoint for the canonical functor $\mathbf{K}(\mathcal{A}) \rightarrow \mathbf{D}(\mathcal{A})$. This implies that $F \circ F_{\rho} \cong \operatorname{Id}_{\mathbf{D}(\mathcal{A})}$. On the other hand, $\mathbf{K}_{\mathrm{ac}}(\operatorname{Inj} \mathcal{A})$ is the kernel of $F$. Thus we conclude that the sequence (3.1) is a localization sequence.

We add some useful remarks which are immediate consequences.

Remark 3.7. Let $J_{\lambda}: \mathbf{K}(\mathcal{A}) \rightarrow \mathbf{K}(\operatorname{Inj} \mathcal{A})$ be the left adjoint of the inclusion $\mathbf{K}(\operatorname{Inj} \mathcal{A}) \rightarrow \mathbf{K}(\mathcal{A})$. Then the composite $Q \circ J_{\lambda}$ is naturally isomorphic to the canonical functor $\mathbf{K}(\mathcal{A}) \rightarrow \mathbf{D}(\mathcal{A})$. 
Remark 3.8. The right adjoint $Q_{\rho}$ of $Q$ induces an equivalence

$$
\mathbf{D}^{\mathrm{b}}(\operatorname{noeth} \mathcal{A}) \stackrel{\sim}{\longrightarrow} \mathbf{K}^{\mathrm{c}}(\operatorname{Inj} \mathcal{A})
$$

which is a quasi-inverse for the equivalence $\mathbf{K}^{\mathrm{c}}(\operatorname{Inj} \mathcal{A}) \rightarrow \mathbf{D}^{\mathrm{b}}($ noeth $\mathcal{A})$ induced by $Q$.

Let us denote by $\mathbf{K}_{\mathrm{inj}}(\mathcal{A})$ the full subcategory of complexes $Y$ in $\mathbf{K}(\operatorname{Inj} \mathcal{A})$ such that $\operatorname{Hom}_{\mathbf{K}(\mathcal{A})}(X, Y)=0$ for all acyclic complexes $X$ in $\mathbf{K}(\mathcal{A})$. Following Spaltenstein's terminology [Spa88], the objects in $\mathbf{K}_{\text {inj }}(\mathcal{A})$ are precisely the $K$-injective complexes having injective components. There are various results about K-injective resolutions in the literature; see for instance [Spa88, BN93]. The following is certainly not the most general; however, it is sufficient in our context.

Corollary 3.9. The inclusion $\mathbf{K}_{\mathrm{inj}}(\mathcal{A}) \rightarrow \mathbf{K}(\mathcal{A})$ has a left adjoint $i: \mathbf{K}(\mathcal{A}) \rightarrow \mathbf{K}_{\mathrm{inj}}(\mathcal{A})$ which has the following properties.

(1) Every object $X$ in $\mathbf{K}(\mathcal{A})$ fits into an exact triangle

$$
a X \longrightarrow X \longrightarrow i X \longrightarrow \Sigma(a X)
$$

such that $a X$ is an acyclic complex.

(2) The functor $i: \mathbf{K}(\mathcal{A}) \rightarrow \mathbf{K}_{\mathrm{inj}}(\mathcal{A})$ induces an equivalence

$$
\mathbf{D}(\mathcal{A})=\mathbf{K}(\mathcal{A}) / \mathbf{K}_{\mathrm{ac}}(\mathcal{A}) \stackrel{\sim}{\longrightarrow} \mathbf{K}_{\mathrm{inj}}(\mathcal{A}) .
$$

(3) We have for all $X, Y$ in $\mathbf{K}(\mathcal{A})$

$$
\operatorname{Hom}_{\mathbf{D}(\mathcal{A})}(X, Y) \cong \operatorname{Hom}_{\mathbf{K}(\mathcal{A})}(X, i Y) .
$$

Proof. Put $i X=Q_{\rho} X$ for each $X$ in $\mathbf{K}(\mathcal{A})$, where $Q_{\rho}$ denotes the right adjoint of $Q: \mathbf{K}(\operatorname{Inj} \mathcal{A}) \rightarrow$ $\mathbf{D}(\mathcal{A})$. The properties of the functor $i$ follow from the fact that $J \circ Q_{\rho}$ is a right adjoint of the canonical functor $\mathbf{K}(\mathcal{A}) \rightarrow \mathbf{D}(\mathcal{A})$. In particular, we see that $i X$ is a K-injective complex.

The functor

$$
R: \mathbf{D}(\mathcal{A})=\mathbf{K}(\mathcal{A}) / \mathbf{K}_{\mathrm{ac}}(\mathcal{A}) \stackrel{\sim}{\longrightarrow} \mathbf{K}_{\mathrm{inj}}(\mathcal{A}) \stackrel{\text { inc }}{\longrightarrow} \mathbf{K}(\mathcal{A})
$$

provides a right adjoint for the canonical functor $\mathbf{K}(\mathcal{A}) \rightarrow \mathbf{D}(\mathcal{A})$. Let us mention as an application that the right derived functor of any additive functor $F: \mathcal{A} \rightarrow \mathcal{B}$ is obtained as composite

$$
\mathbf{R} F: \mathbf{D}(\mathcal{A}) \stackrel{R}{\longrightarrow} \mathbf{K}(\mathcal{A}) \stackrel{\mathbf{K}(F)}{\longrightarrow} \mathbf{K}(\mathcal{B}) \stackrel{\operatorname{can}}{\longrightarrow} \mathbf{D}(\mathcal{B}) .
$$

Example 3.10. Suppose that every object in $\mathcal{A}$ has finite injective dimension. Then the functor $\mathbf{K}(\operatorname{Inj} \mathcal{A}) \rightarrow \mathbf{D}(\mathcal{A})$ is an equivalence since $\mathbf{K}_{\mathrm{ac}}(\operatorname{Inj} \mathcal{A})=0$. In particular, the compact objects in $\mathbf{D}(\mathcal{A})$ are precisely those from $\mathbf{D}^{\mathrm{b}}($ noeth $\mathcal{A})$.

Example 3.11. Suppose that products in $\mathcal{A}$ are exact. For instance, let $\mathcal{A}$ be a module category. Then one can show that $\mathbf{K}_{\text {inj }}(\mathcal{A})$ is the smallest triangulated subcategory of $\mathbf{K}(\mathcal{A})$ which is closed under taking products and contains the injective objects of $\mathcal{A}$ (viewed as complexes concentrated in degree zero).

\section{A recollement}

In this section, we provide a criterion for $\mathcal{A}$ such that the sequence

$$
\mathbf{K}_{\mathrm{ac}}(\operatorname{Inj} \mathcal{A}) \stackrel{I}{\longrightarrow} \mathbf{K}(\operatorname{Inj} \mathcal{A}) \stackrel{Q}{\longrightarrow} \mathbf{D}(\mathcal{A})
$$

induces a recollement

$$
\mathbf{K}_{\mathrm{ac}}(\operatorname{Inj} \mathcal{A}) \rightleftarrows \mathbf{K}(\operatorname{Inj} \mathcal{A}) \rightleftarrows \mathbf{D}(\mathcal{A})
$$




\section{H. KRAUSE}

in the sense of [BBD82]. It is important to note that one cannot expect a recollement

$$
\mathbf{K}_{\mathrm{ac}}(\mathcal{A}) \Longleftarrow \mathbf{K}(\mathcal{A}) \Longleftarrow \mathbf{D}(\mathcal{A})
$$

without severe restrictions on $\mathcal{A}$; see Example 4.9. In fact, a recollement (4.1) implies that a product of exact sequences in $\mathcal{A}$ remains exact.

We begin with a lemma.

Lemma 4.1. Let $\mathcal{A}$ be a locally noetherian Grothendieck category. Then a compact object in $\mathbf{D}(\mathcal{A})$ belongs to $\mathbf{D}^{\mathrm{b}}($ noeth $\mathcal{A})$.

Proof. Suppose that $X$ is compact in $\mathbf{D}(\mathcal{A})$. We need to show that $H^{n} X$ is noetherian for all $n$, and that $H^{n} X$ vanishes for almost all $n$ in $\mathbb{Z}$. We have for any injective object $E$ in $\mathcal{A}$ an isomorphism

$$
\operatorname{Hom}_{\mathbf{D}(\mathcal{A})}(X, E) \cong \operatorname{Hom}_{\mathcal{A}}\left(H^{0} X, E\right) .
$$

Therefore, $\operatorname{Hom}_{\mathcal{A}}\left(H^{0} X,-\right)$ preserves coproducts in $\operatorname{Inj} \mathcal{A}$. This implies that each $H^{n} X$ is noetherian; see [Ren69]. Now fix for each $n$ an injective envelope $H^{n} X \rightarrow E\left(H^{n} X\right)$ and consider the induced map

$$
\alpha: X \longrightarrow \prod_{n \in \mathbb{Z}} \Sigma^{-n} E\left(H^{n} X\right)
$$

in $\mathbf{D}(\mathcal{A})$. The canonical map

$$
\coprod_{n \in \mathbb{Z}} \Sigma^{-n} E\left(H^{n} X\right) \longrightarrow \prod_{n \in \mathbb{Z}} \Sigma^{-n} E\left(H^{n} X\right)
$$

is an isomorphism in $\mathbf{D}(\mathcal{A})$, and therefore $\alpha$ factors though a finite number of factors in

$$
\prod_{n \in \mathbb{Z}} \Sigma^{-n} E\left(H^{n} X\right)
$$

Thus, $H^{n} X$ vanishes for almost all $n$ in $\mathbb{Z}$, and the proof is complete.

We denote by $\mathbf{D}^{\mathrm{c}}(\mathcal{A})$ the full subcategory of $\mathbf{D}(\mathcal{A})$ which is formed by all compact objects.

Theorem 4.2. Let $\mathcal{A}$ be a locally noetherian Grothendieck category and suppose $\mathbf{D}(\mathcal{A})$ is compactly generated. Then the canonical functor $Q: \mathbf{K}(\operatorname{Inj} \mathcal{A}) \rightarrow \mathbf{D}(\mathcal{A})$ has a left adjoint and therefore the sequence

$$
\mathbf{K}_{\mathrm{ac}}(\operatorname{Inj} \mathcal{A}) \stackrel{I}{\longrightarrow} \mathbf{K}(\operatorname{Inj} \mathcal{A}) \stackrel{Q}{\longrightarrow} \mathbf{D}(\mathcal{A})
$$

is a colocalization sequence.

Proof. Let $\mathcal{K}$ be the localizing subcategory of $\mathbf{K}(\operatorname{Inj} \mathcal{A})$ which is generated by all compact objects $X$ in $\mathbf{K}(\operatorname{Inj} \mathcal{A})$ such that $Q X$ is compact in $\mathbf{D}(\mathcal{A})$. We claim that $\left.Q\right|_{\mathcal{K}}: \mathcal{K} \rightarrow \mathbf{D}(\mathcal{A})$ is an equivalence. First note that $\mathcal{K}$ and $\mathbf{D}(\mathcal{A})$ are both compactly generated. We have seen in Lemma 4.1 that

$$
\mathrm{D}^{\mathrm{c}}(\mathcal{A}) \subseteq \mathrm{D}^{\mathrm{b}}(\text { noeth } \mathcal{A})
$$

and $Q$ induces an equivalence

$$
\mathbf{K}^{\mathrm{c}}(\operatorname{Inj} \mathcal{A}) \stackrel{\sim}{\longrightarrow} \mathbf{D}^{\mathrm{b}}(\text { noeth } \mathcal{A}),
$$

by Proposition 2.3. Thus, $Q$ induces an equivalence between the subcategories of compact objects in $\mathcal{K}$ and $\mathbf{D}(\mathcal{A})$. Then a standard argument shows that $\left.Q\right|_{\mathcal{K}}$ is an equivalence since $Q$ preserves all coproducts. Now fix a left adjoint $L: \mathbf{D}(\mathcal{A}) \rightarrow \mathcal{K}$. We claim that the composite

$$
\mathbf{D}(\mathcal{A}) \stackrel{L}{\longrightarrow} \mathcal{K} \stackrel{\operatorname{inc}}{\longrightarrow} \mathbf{K}(\operatorname{Inj} \mathcal{A})
$$


is a left adjoint for $Q$. To see this, consider for objects $X$ in $\mathbf{D}(\mathcal{A})$ and $Y$ in $\mathbf{K}(\operatorname{Inj} \mathcal{A})$ the natural map

$$
\alpha_{X, Y}: \operatorname{Hom}_{\mathbf{K}(\operatorname{Inj} \mathcal{A})}(L X, Y) \longrightarrow \operatorname{Hom}_{\mathbf{D}(\mathcal{A})}(Q L X, Q Y) \stackrel{\sim}{\longrightarrow} \operatorname{Hom}_{\mathbf{D}(\mathcal{A})}(X, Q Y)
$$

which is induced by $Q$. If $X$ and $Y$ are compact, then $\alpha_{X, Y}$ is bijective by Proposition 2.3. We use a standard argument to show that $\alpha_{X, Y}$ is bijective for arbitrary $X$ and $Y$. Fix a compact object $X$. Then the objects $Y$ such that $\alpha_{X, Y}$ is bijective form a triangulated subcategory which is closed under taking coproducts and contains all compact objects. Thus, $\alpha_{X, Y}$ is bijective for all $Y$ because $\mathbf{K}(\operatorname{Inj} \mathcal{A})$ is compactly generated. Now fix any object $Y$. The same argument shows that $\alpha_{X, Y}$ is bijective for all $X$ because $\mathbf{D}(\mathcal{A})$ is compactly generated. We conclude that $Q$ has a left adjoint. Moreover, Lemma 3.2 implies that $I$ and $Q$ form a colocalization sequence.

Following Beilinson et al. [BBD82], we say that a sequence

$$
\mathcal{T}^{\prime} \longrightarrow \mathcal{T} \longrightarrow \mathcal{T}^{\prime \prime}
$$

of exact functors between triangulated categories induces a recollement

$$
\mathcal{T}^{\prime} \rightleftarrows \mathcal{T} \rightleftarrows \mathcal{T}^{\prime \prime}
$$

if the sequence (4.2) is a localization sequence and a colocalization sequence in the sense of Definition 3.1.

Corollary 4.3. Let $\mathcal{A}$ be a locally noetherian Grothendieck category and suppose that $\mathbf{D}(\mathcal{A})$ is compactly generated. Then the sequence

$$
\mathbf{K}_{\mathrm{ac}}(\operatorname{Inj} \mathcal{A}) \stackrel{I}{\longrightarrow} \mathbf{K}(\operatorname{Inj} \mathcal{A}) \stackrel{Q}{\longrightarrow} \mathbf{D}(\mathcal{A})
$$

induces a recollement

$$
\mathbf{K}_{\mathrm{ac}}(\operatorname{Inj} \mathcal{A}) \rightleftarrows \mathbf{K}(\operatorname{Inj} \mathcal{A}) \rightleftarrows \mathbf{D}(\mathcal{A})
$$

Corollary 4.4. Let $\mathcal{A}$ be a locally noetherian Grothendieck category and suppose that $\mathbf{D}(\mathcal{A})$ is compactly generated. Then a product of acyclic complexes of injective objects in $\mathcal{A}$ is acyclic.

Let us give a criterion for $\mathcal{A}$ such that the derived category $\mathbf{D}(\mathcal{A})$ is compactly generated.

Lemma 4.5. Let $\mathcal{A}$ be a locally noetherian Grothendieck category. Suppose that there is a set $\mathcal{A}_{0}$ of objects in $\mathcal{A}$ which are compact when viewed as objects in $\mathbf{D}(\mathcal{A})$. If $\mathcal{A}_{0}$ generates $\mathcal{A}$, then $\mathbf{D}(\mathcal{A})$ is compactly generated by $\mathcal{A}_{0}$.

The lemma is an immediate consequence of the following statement.

Lemma 4.6. Let $\mathcal{A}$ be a locally noetherian Grothendieck category and fix a set $\mathcal{A}_{0}$ of generating objects. Let $X$ be a complex in $\mathcal{A}$ such that $H^{0} X \neq 0$. Then there exists some object $A$ in $\mathcal{A}_{0}$ such that

$$
\operatorname{Hom}_{\mathbf{K}(\mathcal{A})}(A, X) \neq 0 \text { and } \operatorname{Hom}_{\mathbf{D}(\mathcal{A})}(A, X) \neq 0 .
$$

Proof. Choose $A$ in $\mathcal{A}_{0}$ and a map $A \rightarrow Z^{0} X$ such that the composite with $Z^{0} X \rightarrow H^{0} X$ is non-zero. This induces a non-zero element in

$$
H^{0}\left(\operatorname{Hom}_{\mathcal{A}}(A, X)\right) \cong \operatorname{Hom}_{\mathbf{K}(\mathcal{A})}(A, X) .
$$

The second assertion follows from the first since for any object $A$ in $\mathcal{A}$ we have

$$
\operatorname{Hom}_{\mathbf{D}(\mathcal{A})}(A, X) \cong \operatorname{Hom}_{\mathbf{K}(\mathcal{A})}(A, i X)
$$

and $H^{0}(i X) \cong H^{0} X$. 


\section{H. Krause}

We give examples of Grothendieck categories such that objects in $\mathcal{A}$ become compact objects in $\mathbf{D}(\mathcal{A})$.

Example 4.7. Let $\Lambda$ be an associative ring. Denote by $\mathcal{A}=\operatorname{Mod} \Lambda$ the category of (right) $\Lambda$-modules and by $\operatorname{proj} \Lambda$ the full subcategory of finitely generated projective $\Lambda$-modules. Then every object in $\operatorname{proj} \Lambda$ is compact when viewed as object in $\mathbf{D}(\mathcal{A})$. Thus, the inclusion $\mathbf{D}^{\mathrm{b}}(\operatorname{proj} \Lambda) \rightarrow \mathbf{D}(\mathcal{A})$ identifies $\mathbf{D}^{\mathrm{b}}(\operatorname{proj} \Lambda)$ with the full subcategory of compact objects in $\mathbf{D}(\mathcal{A})$. Suppose now that $\Lambda$ is right noetherian. Then the fully faithful functor $Q_{\lambda}: \mathbf{D}(\mathcal{A}) \rightarrow \mathbf{K}(\operatorname{Inj} \mathcal{A})$ identifies $\mathbf{D}(\mathcal{A})$ with the localizing subcategory of $\mathbf{K}(\operatorname{Inj} \mathcal{A})$ which is generated by the injective resolution $i \Lambda$ of $\Lambda$.

Let us return to the completion problem for triangulated categories which has been addressed in Section 2. Keeping the analogy between the completion with respect to filtered colimits and the completion with respect to triangles and coproducts, we obtain the following diagram for a right noetherian ring $\Lambda$. The vertical arrows denote completions and the horizontal arrows denote the appropriate inclusions.
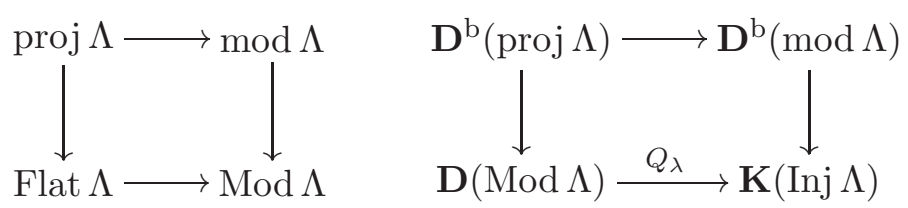

Here, Flat $\Lambda$ denotes the full subcategory of flat $\Lambda$-modules, which is the closure of proj $\Lambda$ under forming filtered colimits.

Example 4.8. Let $\mathbb{X}$ be a quasi-compact and separated scheme, and let $L$ be a locally free sheaf of finite rank. Then

$$
\operatorname{Hom}_{\mathbf{D}(\mathrm{Qcoh} \mathbb{X})}(L,-) \cong \operatorname{Hom}_{\mathbf{D}(\mathrm{Qcoh} \mathbb{X})}\left(\mathcal{O}_{\mathbb{X}}, L^{\vee} \otimes_{\mathcal{O}_{\mathbb{X}}}-\right) \cong H^{0}\left(L^{\vee} \otimes_{\mathcal{O}_{\mathbb{X}}}-\right),
$$

where $L^{\vee}=\mathcal{H} m_{\mathcal{O}_{\mathbb{X}}}\left(L, \mathcal{O}_{\mathbb{X}}\right)$. Thus $L$ is a compact object in $\mathbf{D}($ Qcoh $\mathbb{X})$; see [Nee96]. If $\mathbb{X}$ has an ample family of line bundles, then the locally free sheaves of finite rank generate Qcoh $\mathbb{X}$.

It would be interesting to know in which generality products of acyclic complexes of injectives are acyclic. In fact, the author knows of no examples where this property fails. However, it is important to restrict to complexes of injectives. In order to illustrate this point, let us include an example which shows that products in Qcoh $\mathbb{X}$ need not to be exact. This example was learned from Bernhard Keller.

Example 4.9. Let $k$ be a field and $\mathbb{X}=\mathbb{P}_{k}^{1}$ the projective line with homogeneous coordinate ring $S=k\left[x_{0}, x_{1}\right]$. For each $n \geqslant 0$, we have a canonical map

$$
\pi_{n}: \mathcal{O}(-n) \otimes_{k} \operatorname{Hom}_{\mathbb{X}}(\mathcal{O}(-n), \mathcal{O}) \longrightarrow \mathcal{O}
$$

which is an epimorphism in Qcoh $\mathbb{X}$. We claim that the product

$$
\pi: \prod_{n \geqslant 0}\left(\mathcal{O}(-n) \otimes_{k} \operatorname{Hom}_{\mathbb{X}}(\mathcal{O}(-n), \mathcal{O})\right) \longrightarrow \prod_{n \geqslant 0} \mathcal{O}
$$

is not an epimorphism. Taking graded global sections gives for each $n \geqslant 0$ the multiplication map

$$
\Gamma_{*}\left(\mathbb{X}, \pi_{n}\right): S(-n) \otimes_{k} S_{n} \longrightarrow S
$$

which is a map of graded $S$-modules with cokernel of finite length. However, the cokernel of

$$
\Gamma_{*}(\mathbb{X}, \pi)=\prod_{n \geqslant 0} \Gamma_{*}\left(\mathbb{X}, \pi_{n}\right)
$$


is not a torsion module. The left adjoint of $\Gamma_{*}(\mathbb{X},-)$ is exact and takes $\Gamma_{*}(\mathbb{X}, \pi)$ to $\pi$. It follows that the cokernel of $\pi$ is non-zero, because the left adjoint of $\Gamma_{*}(\mathbb{X},-)$ annihilates exactly those $S$-modules which are torsion modules.

\section{The stable derived category}

Let $\mathcal{A}$ be a locally noetherian Grothendieck category. We suppose that $\mathbf{D}(\mathcal{A})$ is compactly generated.

Definition 5.1. The stable derived category $\mathbf{S}(\mathcal{A})$ of $\mathcal{A}$ is by definition the full subcategory of $\mathbf{K}(\mathcal{A})$ which is formed by all acyclic complexes of injective objects in $\mathcal{A}$. The full subcategory of compact objects is denoted by $\mathbf{S}^{\mathrm{c}}(\mathcal{A})$.

In this section, we show that the stable derived category is compactly generated, and the description of the category of compact objects justifies our terminology. Our basic tool is the (co)localization sequence

$$
\mathbf{S}(\mathcal{A}) \stackrel{I}{\longrightarrow} \mathbf{K}(\operatorname{Inj} \mathcal{A}) \stackrel{Q}{\longrightarrow} \mathbf{D}(\mathcal{A}) .
$$

Thus we use the fact that $I$ and $Q$ have left adjoints $I_{\lambda}, Q_{\lambda}$ and right adjoints $I_{\rho}, Q_{\rho}$. The stabilization functor is by definition the composite

$$
S: \mathbf{D}(\mathcal{A}) \stackrel{I_{\lambda} \circ Q_{\rho}}{\longrightarrow} \mathbf{S}(\mathcal{A}) .
$$

We begin with the following lemma.

Lemma 5.2. Let $\mathcal{A}$ be a locally noetherian Grothendieck category and suppose $\mathbf{D}(\mathcal{A})$ is compactly generated. The functors $Q_{\lambda}, Q_{\rho}: \mathbf{D}(\mathcal{A}) \rightarrow \mathbf{K}(\operatorname{Inj} \mathcal{A})$ admit a natural transformation $\eta: Q_{\lambda} \rightarrow Q_{\rho}$, and $\eta$ is an isomorphism when restricted to the subcategory of compact objects in $\mathbf{D}(\mathcal{A})$.

Proof. We have a natural isomorphism $\mu: \operatorname{Id}_{\mathbf{D}(\mathcal{A})} \stackrel{\sim}{\rightarrow} Q \circ Q_{\lambda}$. The natural transformation

$$
Q_{\lambda} \circ Q \longrightarrow \operatorname{Id}_{\mathbf{K}(\operatorname{Inj} \mathcal{A})} \longrightarrow Q_{\rho} \circ Q
$$

induces for each $X$ in $\mathbf{D}(\mathcal{A})$ a natural map

$$
\eta_{X}: Q_{\lambda} X \stackrel{Q_{\lambda}\left(\mu_{X}\right)}{\longrightarrow}\left(Q_{\lambda} \circ Q\right) Q_{\lambda} X \longrightarrow\left(Q_{\rho} \circ Q\right) Q_{\lambda} X \stackrel{Q_{\rho}\left(\mu_{X}^{-1}\right)}{\longrightarrow} Q_{\rho} X .
$$

Note that $Q(\eta)$ induces an isomorphism

$$
Q \circ Q_{\lambda} \stackrel{\sim}{\longrightarrow} Q \circ Q_{\rho} .
$$

We know from Proposition 2.3 that $Q$ induces an equivalence

$$
\mathbf{K}^{\mathrm{c}}(\operatorname{Inj} \mathcal{A}) \stackrel{\sim}{\longrightarrow} \mathbf{D}^{\mathrm{b}}(\text { noeth } \mathcal{A}) .
$$

On the other hand,

$$
Q_{\lambda}\left(\mathbf{D}^{\mathrm{c}}(\mathcal{A})\right) \subseteq \mathbf{K}^{\mathrm{c}}(\operatorname{Inj} \mathcal{A})
$$

since a left adjoint preserves compactness if the right adjoint preserves coproducts; see Lemma 3.5. Also,

$$
Q_{\rho}\left(\mathbf{D}^{\mathrm{c}}(\mathcal{A})\right) \subseteq \mathbf{K}^{\mathrm{c}}(\operatorname{Inj} \mathcal{A})
$$

since $\mathbf{D}^{\mathrm{c}}(\mathcal{A}) \subseteq \mathbf{D}^{\mathrm{b}}($ noeth $\mathcal{A})$ by Lemma 4.1 , and

$$
Q_{\rho}\left(\mathbf{D}^{\mathrm{b}}(\operatorname{noeth} \mathcal{A})\right)=\mathbf{K}^{\mathrm{c}}(\operatorname{Inj} \mathcal{A})
$$

by Remark 3.8. We conclude that $\left.\eta\right|_{\mathbf{D}^{\mathrm{c}}(\mathcal{A})}$ is an isomorphism. 


\section{H. KRAUSE}

Proposition 5.3. Let $\mathcal{A}$ be a locally noetherian Grothendieck category, and suppose $\mathbf{D}(\mathcal{A})$ is compactly generated. Then we have a localization sequence

$$
\mathbf{D}(\mathcal{A}) \stackrel{Q_{\lambda}}{\longrightarrow} \mathbf{K}(\operatorname{Inj} \mathcal{A}) \stackrel{I_{\lambda}}{\longrightarrow} \mathbf{S}(\mathcal{A})
$$

which induces the following commutative diagram.

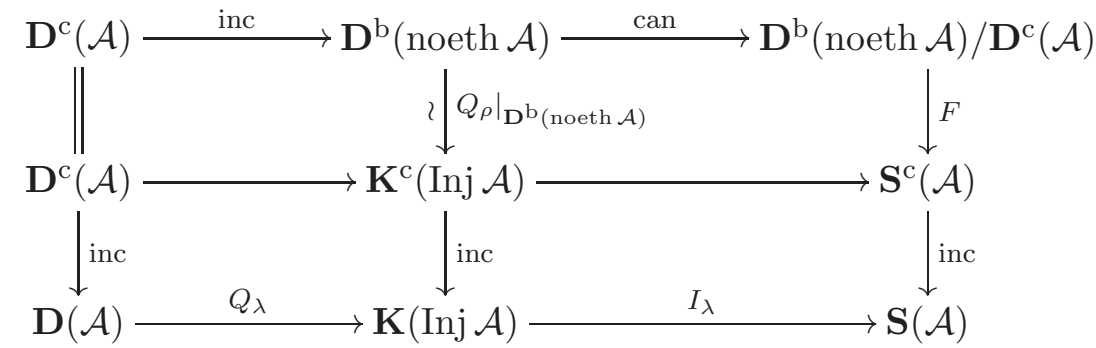

Proof. It follows from Theorem 4.2 that the sequence (5.1) is a localization sequence. Let us explain the commutativity of the diagram. First observe that a left adjoint preserves compactness if the right adjoint preserves coproducts; see Lemma 3.5. Therefore, $I_{\lambda}$ and $Q_{\lambda}$ preserve compactness, and this explains the commutativity of the lower squares. Now observe that

$$
\mathbf{D}^{\mathrm{c}}(\mathcal{A}) \subseteq \mathbf{D}^{\mathrm{b}}(\text { noeth } \mathcal{A})
$$

by Lemma 4.1, and that $\left.Q_{\rho}\right|_{\mathbf{D}^{\mathrm{b}}(\text { noeth } \mathcal{A})}$ is a quasi-inverse for $\left.Q\right|_{\mathbf{K}^{\mathrm{c}}(\operatorname{Inj} \mathcal{A})}$. It follows from Lemma 5.2 that the upper left-hand square commutes. The functor $F$ is by definition the unique functor making the upper right-hand square commutative. It exists because $I_{\lambda} \circ Q_{\lambda}=0$.

We have seen that the stable derived category $\mathbf{S}(\mathcal{A})$ is a localization of the homotopy category $\mathbf{K}(\operatorname{Inj} \mathcal{A})$. This has some interesting consequences.

Corollary 5.4. The stable derived category $\mathbf{S}(\mathcal{A})$ is compactly generated, and the functor $I_{\lambda} \circ Q_{\rho}: \mathbf{D}(\mathcal{A}) \rightarrow \mathbf{S}(\mathcal{A})$ induces (up to direct factors) an equivalence

$$
F: \mathbf{D}^{\mathrm{b}}(\operatorname{noeth} \mathcal{A}) / \mathbf{D}^{\mathrm{c}}(\mathcal{A}) \stackrel{\sim}{\longrightarrow} \mathbf{S}^{\mathrm{c}}(\mathcal{A}) .
$$

Proof. We know from Proposition 2.3 that $\mathbf{K}(\operatorname{Inj} \mathcal{A})$ is compactly generated. This property carries over to $\mathbf{S}(\mathcal{A})$ since $I_{\lambda}$ sends a set of compact generators of $\mathbf{K}(\operatorname{Inj} \mathcal{A})$ to a set of compact generators of $\mathbf{S}(\mathcal{A})$. The functor $Q_{\lambda}$ identifies $\mathbf{D}(\mathcal{A})$ with the localizing subcategory of $\mathbf{K}(\operatorname{Inj} \mathcal{A})$ which is generated by all compact objects in the image of $Q_{\lambda}$. Now apply the localization theorem of NeemanRavenel-Thomason-Trobaugh-Yao [Nee92]. This result describes the category of compact objects of the quotient $\mathbf{S}(\mathcal{A})$, up to direct factors, as the quotient of the compact objects in $\mathbf{K}(\operatorname{Inj} \mathcal{A})$ modulo those from the localizing subcategory. To be precise, $F$ is fully faithful and every object in $\mathbf{S}^{\mathrm{c}}(\mathcal{A})$ is a direct factor of some object in the image of $F$.

Corollary 5.5. The composite

$$
\mathcal{A} \stackrel{\mathrm{can}}{\longrightarrow} \mathbf{D}(\mathcal{A}) \stackrel{I_{\lambda} \circ Q_{\rho}}{\longrightarrow} \mathbf{S}(\mathcal{A})
$$

preserves all coproducts and annihilates the objects in $\mathcal{A} \cap \mathbf{D}^{\mathrm{c}}(\mathcal{A})$.

Proof. The diagram in Proposition 5.3 shows that $I_{\lambda} \circ Q_{\rho}$ annihilates $\mathcal{A} \cap \mathbf{D}^{\mathrm{c}}(\mathcal{A})$. To show that $I_{\lambda} \circ Q_{\rho}$ preserves all coproducts, observe that $Q_{\rho}$ sends an object in $\mathcal{A}$ to an injective resolution. A coproduct of injective resolutions is again an injective resolution, and the left adjoint $I_{\lambda}$ preserves all coproducts. This finishes the proof. 
Using the stabilization functor $S: \mathbf{D}(\mathcal{A}) \rightarrow \mathbf{S}(\mathcal{A})$, we define for objects $X, Y$ in $\mathbf{D}(\mathcal{A})$ and $n \in \mathbb{Z}$ the stable cohomology group

$$
\underline{\operatorname{Ext}}_{\mathcal{A}}^{n}(X, Y)=\operatorname{Hom}_{\mathbf{K}(\mathcal{A})}\left(S X, \Sigma^{n}(S Y)\right) .
$$

Note that in both arguments, each exact sequence in $\mathcal{A}$ induces a long exact sequence in stable cohomology. We do not go into detail, but refer to our discussion of Tate cohomology in $\S 7$. In fact, both cohomology theories coinciding in case $\mathcal{A}$ satisfies some appropriate Gorenstein property, and we shall see explicit formulae for the Tate cohomology groups.

Example 5.6. Suppose that $\mathcal{A}$ is a module category. Then the stabilization functor annihilates all finitely generated projective modules, and all coproducts of such, by Corollary 5.5. Hence, it annihilates all projective modules. Since $I_{\lambda} \circ Q_{\rho}$ is an exact functor vanishing on projectives, it annihilates all bounded complexes of projective modules. In particular, all modules of finite projective dimension are annihilated. Similarly, if $\mathcal{A}$ is a category of quasi-coherent sheaves, then the stabilization functor annihilates all sheaves having a finite resolution with locally free sheaves.

Given a noetherian scheme $\mathbb{X}$, the stable derived category $\mathbf{S}($ Qcoh $\mathbb{X})$ vanishes if $\mathbb{X}$ is regular. Nonetheless, a classical result of Bernstein et al. [BGG78] shows that stable derived categories are relevant when one studies regular schemes. This is sketched in the following example.

Example 5.7. Let $\Lambda$ be a Koszul algebra and $\Lambda^{!}$its Koszul dual. Then, under appropriate assumptions, we have an equivalence $\mathbf{K}(\operatorname{Inj} \Lambda) \stackrel{\sim}{\rightarrow} \mathbf{K}\left(\operatorname{Inj} \Lambda^{!}\right)$which induces an equivalence $\mathbf{D}^{\mathrm{b}}(\bmod \Lambda) \stackrel{\sim}{\rightarrow}$ $\mathbf{D}^{\mathrm{b}}\left(\bmod \Lambda^{!}\right)$when restricted to the full subcategories of compact objects [BGS96, Kel94]. Note that we consider the categories of graded modules over $\Lambda$ and $\Lambda^{!}$, respectively. The classical example is the symmetric algebra $\Lambda=S V$ of a $d$-dimensional space $V$ over a field $k$, where $\Lambda^{!}$is the exterior algebra $\Lambda V^{*}$ of the dual space $V^{*}$. The equivalence $\mathbf{K}(\operatorname{Inj} \Lambda) \stackrel{\sim}{\rightarrow} \mathbf{K}\left(\operatorname{Inj} \Lambda^{!}\right)$takes an injective resolution $i k$ of $\Lambda_{0}=k$ to $\Lambda^{!}$and identifies the localizing subcategory $\mathcal{K}$ generated by $i k$ with the localizing subcategory generated by $\Lambda^{!}$, which is $\mathbf{D}\left(\operatorname{Mod} \Lambda^{!}\right)$. Note that the quotient $\mathbf{K}(\operatorname{Inj} \Lambda) / \mathcal{K}$ identifies with the derived category of the quotient $\operatorname{Mod} \Lambda /(\operatorname{Mod} \Lambda)_{0}$, where $(\operatorname{Mod} \Lambda)_{0}$ denotes the subcategory of torsion modules. This quotient is equivalent to Qcoh $\mathbb{P}_{k}^{d-1}$ by Serre's Theorem. Thus, we obtain an equivalence

$$
\mathbf{D}\left(\mathrm{Q} \operatorname{coh} \mathbb{P}_{k}^{d-1}\right) \stackrel{\sim}{\longrightarrow} \mathbf{S}\left(\operatorname{Mod} \wedge k^{d}\right) .
$$

Note that $\mathbf{S}\left(\operatorname{Mod} \wedge k^{d}\right)$ is equivalent to the stable module category $\underline{\operatorname{Mod}} \wedge k^{d}$ because the exterior algebra is self-injective; see Example 7.16. Passing to the subcategory of compact objects, one obtains the equivalence

$$
\mathbf{D}^{\mathrm{b}}\left(\operatorname{coh} \mathbb{P}_{k}^{d-1}\right) \stackrel{\sim}{\longrightarrow} \underline{\bmod } \wedge k^{d}
$$

of Bernstein et al. [BGG78], where $\underline{\bmod } \wedge k^{d}$ denotes the stable category of all finite-dimensional $\wedge k^{d}$-modules. This example generalizes to non-commutative algebras, for instance, to Artin-Schelter regular algebras [Jor03].

\section{Extending derived functors}

An additive functor $F: \mathcal{A} \rightarrow \mathcal{B}$ between locally noetherian Grothendieck categories admits a right derived functor $\mathbf{R} F: \mathbf{D}(\mathcal{A}) \rightarrow \mathbf{D}(\mathcal{B})$. In this section, we extend this to a functor $\hat{\mathbf{R}} F: \mathbf{K}(\operatorname{Inj} \mathcal{A}) \rightarrow$ $\mathbf{K}(\operatorname{Inj} \mathcal{B})$ and investigate its right and left adjoints. As an application, we consider for $F$ the direct image functor $f_{*}:$ Qcoh $\mathbb{X} \rightarrow$ Qcoh $\mathbb{Y}$ corresponding to a morphism $f: \mathbb{X} \rightarrow \mathbb{Y}$ between noetherian schemes. We use the following functors

$$
J: \mathbf{K}(\operatorname{Inj} \mathcal{A}) \stackrel{\text { inc }}{\longrightarrow} \mathbf{K}(\mathcal{A}) \quad \text { and } \quad Q: \mathbf{K}(\operatorname{Inj} \mathcal{A}) \stackrel{\text { inc }}{\longrightarrow} \mathbf{K}(\mathcal{A}) \stackrel{\text { can }}{\longrightarrow} \mathbf{D}(\mathcal{A})
$$




\section{H. KRAUSE}

simultaneously for $\mathcal{A}$ and $\mathcal{B}$. Moreover, we use the fact that both functors have left and right adjoints.

Theorem 6.1. Let $F: \mathcal{A} \rightarrow \mathcal{B}$ be an additive functor between locally noetherian Grothendieck categories. Suppose that $\mathbf{D}(\mathcal{A})$ and $\mathbf{D}(\mathcal{B})$ are compactly generated. Then the composite

$$
\hat{\mathbf{R}} F: \mathbf{K}(\operatorname{Inj} \mathcal{A}) \stackrel{J}{\longrightarrow} \mathbf{K}(\mathcal{A}) \stackrel{\mathbf{K}(F)}{\longrightarrow} \mathbf{K}(\mathcal{B}) \stackrel{J_{\lambda}}{\longrightarrow} \mathbf{K}(\operatorname{Inj} \mathcal{B})
$$

makes the following diagram commutative.

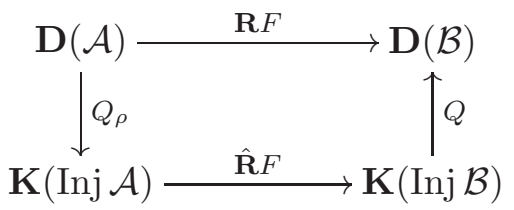

(1) Suppose $F$ preserves coproducts. Then $\hat{\mathbf{R}} F$ preserves coproducts and therefore has a right adjoint $(\hat{\mathbf{R}} F)_{\rho}$.

(2) Suppose $F$ and $\mathbf{R} F$ preserve coproducts. Then $\mathbf{R} F$ has a right adjoint $(\mathbf{R} F)_{\rho}$ making the following diagrams commutative.
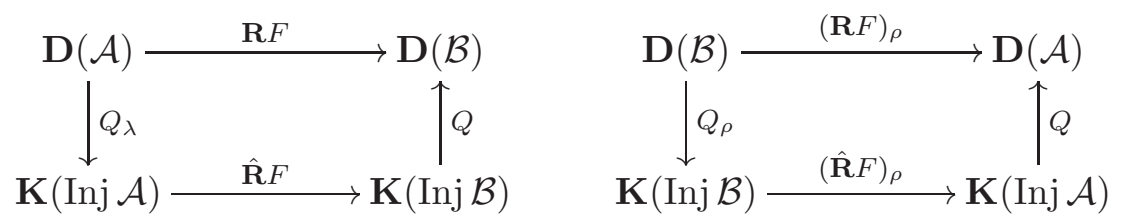

Proof. The composite

$$
\mathbf{K}(\mathcal{A}) \stackrel{J_{\lambda}}{\longrightarrow} \mathbf{K}(\operatorname{Inj} \mathcal{A}) \stackrel{Q}{\longrightarrow} \mathbf{D}(\mathcal{A})
$$

is naturally isomorphic to the canonical functor $\mathbf{K}(\mathcal{A}) \rightarrow \mathbf{D}(\mathcal{A})$; see Remark 3.7. Clearly, $J \circ Q_{\rho}$ is its right adjoint. We denote by $\mathbf{R} F$ the right derived functor of $F$ and have

$$
\mathbf{R} F=Q \circ J_{\lambda} \circ \mathbf{K}(F) \circ J \circ Q_{\rho} .
$$

Using the definition $\hat{\mathbf{R}} F=J_{\lambda} \circ \mathbf{K}(F) \circ J$, we obtain $\mathbf{R} F=Q \circ \hat{\mathbf{R}} F \circ Q_{\rho}$.

(1) Suppose that $F$ preserves coproducts. Then $\mathbf{K}(F)$ preserves coproducts. It follows that $\hat{\mathbf{R}} F$ preserves coproducts since $J$ and $J_{\lambda}$ preserve coproducts. Now apply Proposition 3.3 to obtain a right adjoint for $\hat{\mathbf{R}} F$.

(2) Suppose that $F$ and $\mathbf{R} F$ preserve coproducts. Then $\mathbf{R} F$ has a right adjoint by Proposition 3.3. Next we show that

$$
Q \circ \hat{\mathbf{R}} F \circ Q_{\lambda} \cong Q \circ \hat{\mathbf{R}} F \circ Q_{\rho} .
$$

We have a natural transformation $Q_{\lambda} \rightarrow Q_{\rho}$ which is induced from the natural transformation $Q_{\lambda} \circ Q \rightarrow Q_{\rho} \circ Q$. Now apply $Q \circ \hat{\mathbf{R}} F$ to get a natural transformation

$$
\mu: Q \circ \hat{\mathbf{R}} F \circ Q_{\lambda} \longrightarrow Q \circ \hat{\mathbf{R}} F \circ Q_{\rho} .
$$

It is shown in Lemma 5.2 that $Q_{\lambda} \rightarrow Q_{\rho}$ is an isomorphism when restricted to compact objects in $\mathbf{D}(\mathcal{A})$. On the other hand, $Q \circ \hat{\mathbf{R}} F \circ Q_{\lambda}$ and $Q \circ \hat{\mathbf{R}} F \circ Q_{\rho}$ both preserve coproducts by our assumption on $\mathbf{R} F$. It follows that $\mu$ is an isomorphism since $\mathbf{D}(\mathcal{A})$ is compactly generated. Clearly, $Q \circ(\hat{\mathbf{R}} F)_{\rho} \circ Q_{\rho}$ is a right adjoint for $Q \circ \hat{\mathbf{R}} F \circ Q_{\lambda}$. This completes the proof.

The extended derived functor and its right adjoint admit some alternative description. The author is indebted to Bernhard Keller for providing this remark. 
Remark 6.2. It is possible to express $\hat{\mathbf{R}} F$ as the tensor functor and its right adjoint $(\hat{\mathbf{R}} F)_{\rho}$ as the Hom functor with respect to a bimodule of DG categories; see [Kel94, § 6.4]. This depends on the appropriate choice of DG categories $\mathcal{A}_{0}$ and $\mathcal{B}_{0}$ such that $\mathbf{K}(\operatorname{Inj} \mathcal{A}) \cong \mathbf{D}_{\operatorname{dg}}\left(\mathcal{A}_{0}\right)$ and $\mathbf{K}(\operatorname{Inj} \mathcal{B}) \cong$ $\mathbf{D}_{\mathrm{dg}}\left(\mathcal{B}_{0}\right)$, respectively.

Next we consider the following diagram

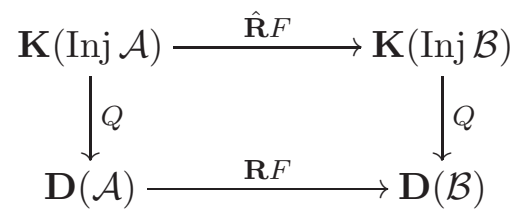

and ask when it is commutative.

Lemma 6.3. Keep the assumptions from Theorem 6.1. There is a natural transformation $Q \circ \hat{\mathbf{R}} F \rightarrow$ $\mathbf{R} F \circ Q$ which is an isomorphism if and only if $F$ sends every acyclic complex of injective objects to an acyclic complex.

Proof. We apply the localization sequence

$$
\mathbf{K}_{\mathrm{ac}}(\operatorname{Inj} \mathcal{A}) \stackrel{I}{\longrightarrow} \mathbf{K}(\operatorname{Inj} \mathcal{A}) \stackrel{Q}{\longrightarrow} \mathbf{D}(\mathcal{A})
$$

from Proposition 3.6. Let $X$ be an object in $\mathbf{K}(\operatorname{Inj} \mathcal{A})$ and consider the triangle

$$
\left(I \circ I_{\rho}\right) X \longrightarrow X \longrightarrow\left(Q_{\rho} \circ Q\right) X \longrightarrow \Sigma\left(I \circ I_{\rho}\right) X
$$

in $\mathbf{K}(\operatorname{Inj} \mathcal{A})$. Now apply $Q \circ \hat{\mathbf{R}} F$ which gives a map

$$
(Q \circ \hat{\mathbf{R}} F) X \longrightarrow(\mathbf{R} F \circ Q) X
$$

since $Q \circ \hat{\mathbf{R}} F \circ Q_{\rho} \cong \mathbf{R} F$, by Theorem 6.1. Clearly, this map is an isomorphism if and only if $Q \circ \hat{\mathbf{R}} F$ annihilates $\left(I \circ I_{\rho}\right) X$.

We include a simple example which illustrates the preceding lemma.

Example 6.4. Let $k$ be a field and $\Lambda=k[t] /\left(t^{2}\right)$. We take the functor

$$
F: \operatorname{Mod} \Lambda \longrightarrow \operatorname{Mod} k, \quad X \mapsto \operatorname{Hom}_{\Lambda}(k, X),
$$

and observe that the following diagram does not commute.

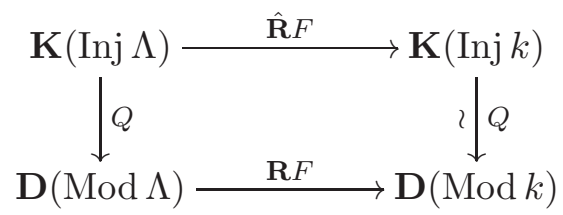

For instance, we have $Q X=0$ and $(\hat{\mathbf{R}} F) X \neq 0$ if we take for $X$ the acyclic complex

$$
\cdots \stackrel{t}{\longrightarrow} \Lambda \stackrel{t}{\longrightarrow} \Lambda \stackrel{t}{\longrightarrow} \Lambda \stackrel{t}{\longrightarrow} \cdots
$$

$\operatorname{in} \mathbf{K}(\operatorname{Inj} \Lambda)$.

Now we specialize and consider as an example a morphism $f: \mathbb{X} \rightarrow \mathbb{Y}$ between separated noetherian schemes. Let $f_{*}:$ Qcoh $\mathbb{X} \rightarrow$ Qcoh $\mathbb{Y}$ denote the direct image functor. Note that the right derived functor $\mathbf{R} f_{*}: \mathbf{D}($ Qcoh $\mathbb{X}) \rightarrow \mathbf{D}($ Qcoh $\mathbb{Y})$ preserves coproducts [Nee96, Lemma 1.4]. Thus, $\mathbf{R} f_{*}$ and its right adjoint Grothendieck duality functor $f^{!}$extend to functors between $\mathbf{K}(\operatorname{Inj} \mathbb{X})$ and $\mathbf{K}(\operatorname{Inj} \mathbb{Y})$, by Theorem 6.1. This is the statement of Theorem 1.4 from the introduction. In fact, in this case 


\section{H. KRAUSE}

the situation is much nicer. The author is grateful to Amnon Neeman for pointing out that the functor $\hat{\mathbf{R}} f_{*}$ and its right adjoint $\left(\hat{\mathbf{R}} f_{*}\right)_{\rho}$ make the following diagrams commutative.
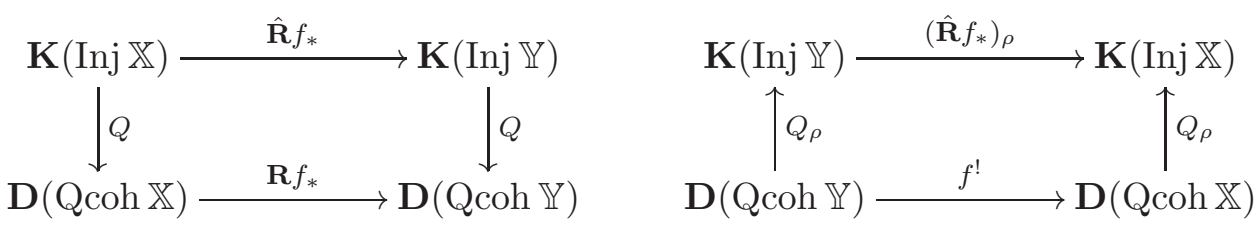

This is essentially the statement of Theorem 1.5 from the introduction. The proof which is due to Neeman goes as follows.

Proof of Theorem 1.5. We need to show that both squares in (6.1) commute. Then we use the localization sequence

$$
\mathbf{S}(\mathrm{Q} \operatorname{coh} \mathbb{X}) \stackrel{I}{\longrightarrow} \mathbf{K}(\operatorname{Inj} \mathbb{X}) \stackrel{Q}{\longrightarrow} \mathbf{D}(Q \operatorname{coh} \mathbb{X})
$$

from Proposition 3.6 and obtain from $\hat{\mathbf{R}} f_{*}$ and $\left(\hat{\mathbf{R}} f_{*}\right)_{\rho}$ an adjoint pair of functors between $\mathbf{S}(\mathrm{Qcoh} \mathbb{X})$ and $\mathbf{S}($ Qcoh $\mathbb{Y})$.

In order to show the commutativity of (6.1), we apply Lemma 6.3 and need to show that $f_{*}$ sends an acyclic complex $X$ of injective objects to an acyclic complex. The question is local in $\mathbb{Y}$ and we may assume $\mathbb{Y}$ affine. Cover $\mathbb{X}$ by a finite number of affines. Then $f_{*}$ can be computed using the Čech cohomology of the cover. If there are $n$ open sets in the cover, then for any quasi-coherent sheaf $A$ we have

$$
\mathbf{R}^{n+1} f_{*} A=0 .
$$

Now take our acyclic complex $X$ of injective sheaves on $\mathbb{X}$. Then the sequence

$$
0 \longrightarrow X^{0} \longrightarrow X^{1} \longrightarrow X^{2} \longrightarrow \cdots
$$

is an injective resolution of the kernel $A$ of the map $X^{0} \rightarrow X^{1}$. Applying $f_{*}$, the sequence computes $\mathbf{R}^{i} f_{*} A$ for us, which vanishes if $i \geqslant n+1$. Thus, $f_{*} X$ is acyclic above degree $n$, but by shifting we conclude that it is acyclic everywhere.

Having shown the commutativity of the left-hand square, the commutativity of the right-hand square follows, because it is obtained by taking right adjoints. Thus, the proof is complete.

Next we investigate for an exact functor $F: \mathcal{A} \rightarrow \mathcal{B}$ an extension $\hat{\mathbf{L}} F$ of the derived functor $\mathbf{L} F: \mathbf{D}(\mathcal{A}) \rightarrow \mathbf{D}(\mathcal{B})$. For this we need some assumptions, and it is convenient to introduce the following notation. As before, $\mathcal{A}$ and $\mathcal{B}$ denote locally noetherian Grothendieck categories. Let $f:$ noeth $\mathcal{A} \rightarrow$ noeth $\mathcal{B}$ be an additive functor. Then there is, up to isomorphism, a unique functor $f^{*}: \mathcal{A} \rightarrow \mathcal{B}$ which extends $f$ and preserves filtered colimits. This has a right adjoint $f_{*}: \mathcal{B} \rightarrow \mathcal{A}$ if and only if $f$ is right exact. Note that $f$ is exact if and only if $f^{*}$ is exact if and only if $f_{*}$ sends injective objects to injective objects. We now give an example.

Example 6.5. Let $f: \mathbb{X} \rightarrow \mathbb{Y}$ be a morphism between noetherian schemes. Then the inverse image functor $f^{*}:$ Qcoh $\mathbb{Y} \rightarrow$ Qcoh $\mathbb{X}$ sends coherent sheaves to coherent sheaves and preserves filtered colimits. Moreover, the direct image functor $f_{*}$ is a right adjoint of $f^{*}$. Our notation is therefore consistent if we identify the morphism $f: \mathbb{X} \rightarrow \mathbb{Y}$ with the functor $\operatorname{coh} \mathbb{Y} \rightarrow \operatorname{coh} \mathbb{X}$.

Theorem 6.6. Let $\mathcal{A}$ and $\mathcal{B}$ locally noetherian Grothendieck categories such that $\mathbf{D}(\mathcal{A})$ and $\mathbf{D}(\mathcal{B})$ are compactly generated. Let $f: \operatorname{noeth} \mathcal{A} \rightarrow \operatorname{noeth} \mathcal{B}$ be an exact functor. Then $\hat{\mathbf{R}} f_{*}$ has a left 
adjoint $\hat{\mathbf{L}} f^{*}$ which induces a functor $\mathbf{S} f^{*}$ making the following diagram commutative.

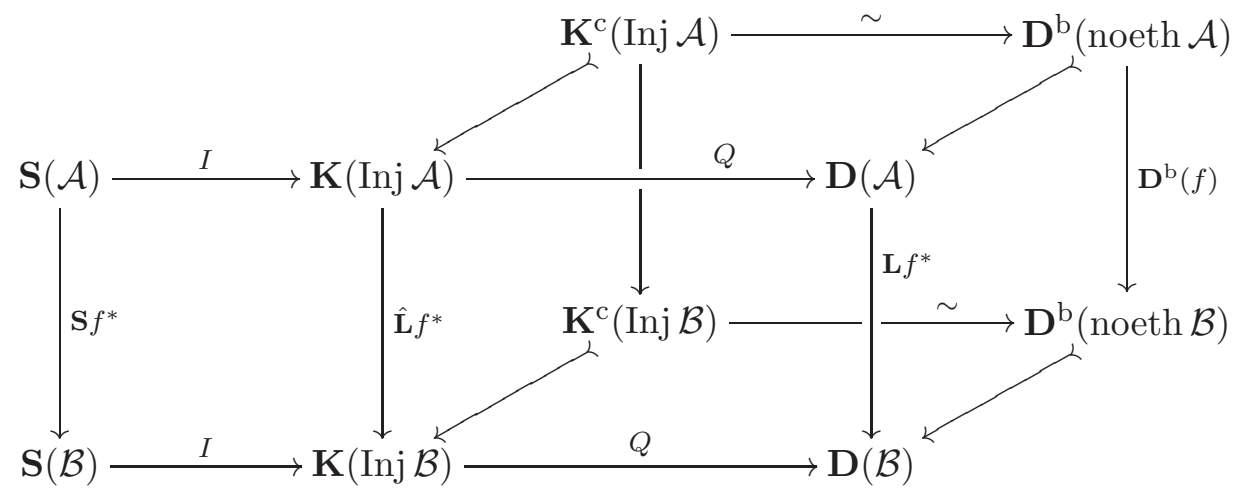

If $\mathbf{R} f_{*}$ preserves coproducts, then, in addition, the following diagram commutes.

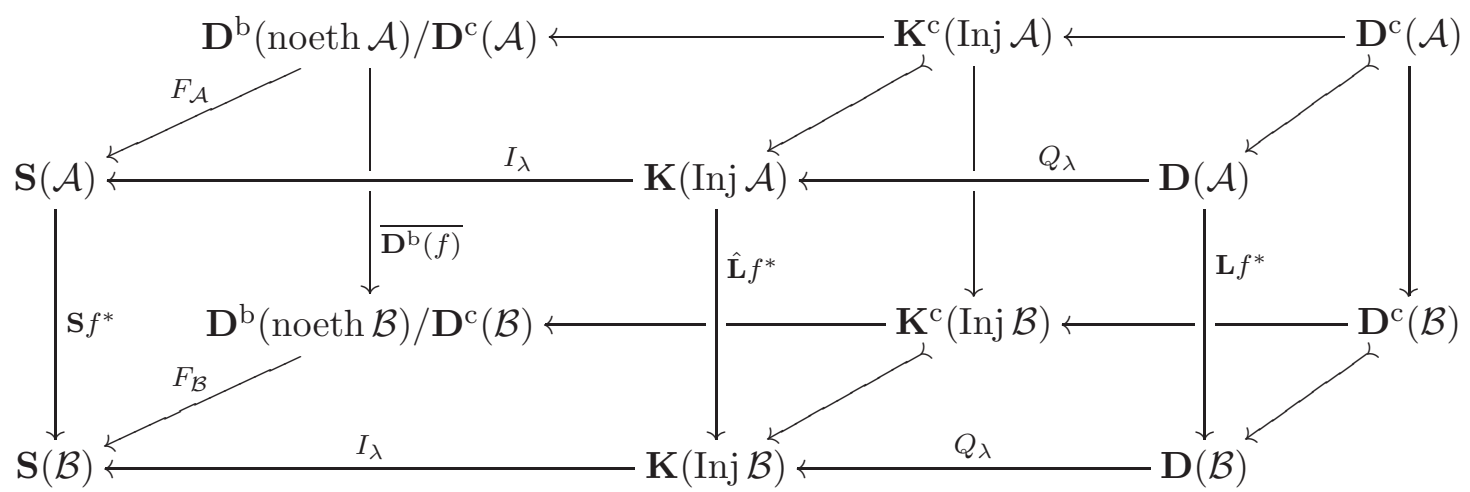

Note that $F_{\mathcal{A}}$ and $F_{\mathcal{B}}$ induce, up to direct factors, equivalences onto the full subcategories of compact objects in $\mathbf{S}(\mathcal{A})$ and $\mathbf{S}(\mathcal{B})$ respectively. Thus, $\overline{\mathbf{D}^{\mathrm{b}}(f)}$ determines the functor $\mathbf{S} f^{*}$.

Proof. The exactness of $f$ implies the exactness of $f^{*}$. Thus, $f_{*}$ sends injective objects to injective objects and we have the following commutative diagram.

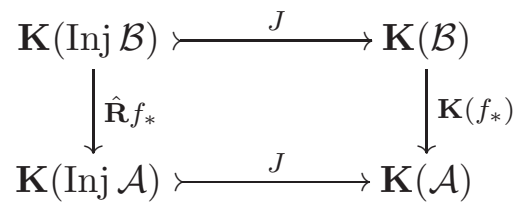

The right adjoint $f_{*}$ preserves products and we therefore have a left adjoint for $\hat{\mathbf{R}} f_{*}$, by Proposition 3.3, which we denote by $\hat{\mathbf{L}} f^{*}$. We obtain the following diagram

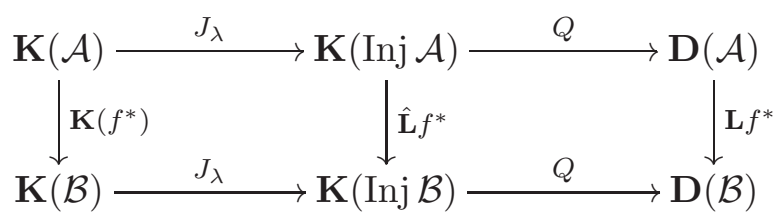

and claim it is commutative. The left-hand square commutes because it is obtained from (6.3) by taking left adjoints. The outer square commutes because the composite $Q \circ J_{\lambda}$ is naturally isomorphic to the canonical functor $\mathbf{K}(\mathcal{A}) \rightarrow \mathbf{D}(\mathcal{A})$; see Remark 3.7. We conclude the commutativity of the right-hand square using that $J_{\lambda} \circ J \cong \operatorname{Id}_{\mathbf{K}(\operatorname{Inj} \mathcal{A})}$. Clearly, $\hat{\mathbf{L}} f^{*}$ sends acyclic complexes to acyclic complexes and we obtain the functor $\mathbf{S} f^{*}$ making the diagram (6.2) commutative. Finally, observe that $\hat{\mathbf{L}} f^{*}$ preserve compactness because its right adjoint $\hat{\mathbf{R}} f_{*}$ preserve coproducts; see Lemma 3.5. Thus, every square in the diagram (6.2) commutes. 


\section{H. KRAUSE}

Now assume in addition that $\mathbf{R} f_{*}$ preserves coproducts. We use again the fact that a left adjoint preserves compactness if the right adjoint preserves coproducts; see Lemma 3.5 . Thus, $\mathbf{L} f^{*}$ and $\hat{\mathbf{L}} f^{*}$ preserve compactness. Note that $Q_{\lambda}$ identifies $\mathbf{D}(\mathcal{A})$ with the localizing subcategory of $\mathbf{K}(\operatorname{Inj} \mathcal{A})$ which is generated by

$$
\mathbf{D}^{\mathrm{c}}(\mathcal{A}) \subseteq \mathbf{D}^{\mathrm{b}}(\text { noeth } \mathcal{A}) \cong \mathbf{K}^{\mathrm{c}}(\operatorname{Inj} \mathcal{A}) \subseteq \mathbf{K}(\operatorname{Inj} \mathcal{A}) .
$$

Of course, the same applies for $\mathcal{B}$. We obtain the following diagram

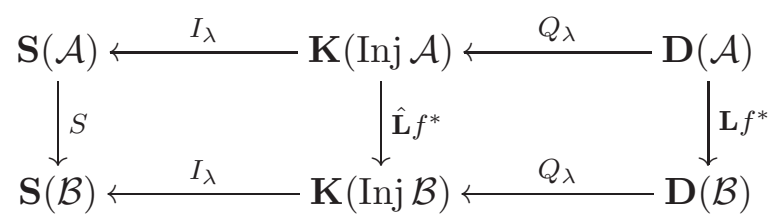

where the right-hand square commutes. The horizontal sequences are localization sequences by Theorem 4.2, and $\hat{\mathbf{L}} f^{*}$ induces a functor $S: \mathbf{S}(\mathcal{A}) \rightarrow \mathbf{S}(\mathcal{B})$ making the left-hand square commutative. Moreover, we have

$$
S=S \circ I_{\lambda} \circ I=I_{\lambda} \circ \hat{\mathbf{L}} f^{*} \circ I=I_{\lambda} \circ I \circ \mathbf{S} f^{*}=\mathbf{S} f^{*} .
$$

The functors $F_{\mathcal{A}}$ and $F_{\mathcal{B}}$ are both induced by $I_{\lambda}$, and the commutativity

$$
\mathbf{S} f^{*} \circ F_{\mathcal{A}}=F_{\mathcal{B}} \circ \overline{\mathbf{D}^{\mathrm{b}}(f)}
$$

is easily checked; see Corollary 5.4. This completes the proof.

The author is grateful to the referee for pointing out possible generalizations.

Remark 6.7. Let $f: \mathbb{X} \rightarrow \mathbb{Y}$ be a morphism between noetherian schemes and suppose that $f^{*}$ is exact. Then $\mathbf{S} f^{*}$ is the left adjoint of $\mathbf{S} f_{*}$ which appears in Theorem 1.5. In fact, this theorem suggests that parts of Theorem 6.6 can be generalized. For instance, the right-hand square in diagram (6.4) does not need any assumption on the morphism $f$ because it is simply the left adjoint of a commutative square in Theorem 1.5.

Next we investigate the inclusion $f: \mathbb{X} \rightarrow \mathbb{Y}$ of an open subscheme. In this case, the adjoint pair of functors $f_{*}$ and $f^{*}$ between Qcoh $\mathbb{X}$ and Qcoh $\mathbb{Y}$ restricts to an adjoint pair of functors between $\operatorname{Inj} \mathbb{X}$ and Inj $\mathbb{Y}$; see $[$ Gabb62, $\S \mathrm{VI}]$. Moreover, $f^{*} \circ f_{*} \cong \operatorname{Id}$ Qcoh $\mathbb{X}$. Thus, we can identify $\hat{\mathbf{R}} f_{*}=f_{*}$ and $\hat{\mathbf{L}} f^{*}=f^{*}$. Note that both functors send acyclic complexes with injective components to acyclic complexes. This is clear for $f^{*}$ because it is exact, and follows for $f_{*}$ from Theorem 1.5, or by looking at the right adjoint of the right-hand square in diagram (6.4). For each sheaf $A$ in Qcoh $\mathbb{Y}$ we denote by $\operatorname{Supp} A$ the support of $A$ and observe that $f^{*}$ annihilates $A$ if and only $\operatorname{Supp} A$ is contained in $\mathbb{Y} \backslash \mathbb{X}$. In fact, the natural map $A \rightarrow\left(f_{*} \circ f^{*}\right) A$ induces a split exact sequence

$$
0 \longrightarrow A^{\prime} \longrightarrow A \longrightarrow\left(f_{*} \circ f^{*}\right) A \longrightarrow 0
$$

if $A$ is injective. In particular, the support of $A^{\prime}$ is contained in $\mathbb{Y} \backslash \mathbb{X}$, whereas the support of $\left(f_{*} \circ f^{*}\right) A$ is contained in $\mathbb{X}$.

Now fix a complex $X$ in $\mathbf{K}(\operatorname{Inj} \mathbb{Y})$. The support of $X$ is by definition

$$
\operatorname{Supp} X=\bigcup_{n \in \mathbb{Z}} \operatorname{Supp} X^{n}
$$

where $X$ is assumed to be homotopically minimal; see Proposition B.2. We write $X_{\mathbb{X}}=\left(f_{*} \circ f^{*}\right) X$, and the natural map $X \rightarrow X_{\mathbb{X}}$ induces an exact triangle

$$
X_{\mathbb{Y} \backslash \mathbb{X}} \longrightarrow X \longrightarrow X_{\mathbb{X}} \longrightarrow \Sigma\left(X_{\mathbb{Y} \backslash \mathbb{X}}\right)
$$

in $\mathbf{K}(\operatorname{Inj} \mathbb{Y})$ where the support of $X_{\mathbb{Y} \backslash \mathbb{X}}$ is contained in $\mathbb{Y} \backslash \mathbb{X}$. 
Lemma 6.8. Let $\mathbb{Y}$ be a separated noetherian scheme and $f: \mathbb{X} \rightarrow \mathbb{Y}$ be the inclusion of an open subscheme. If $X$ is a complex in $\mathbf{K}(\operatorname{Inj} \mathbb{Y})$, then $f^{*} X=0$ if and only if the support of $X$ is contained in $\mathbb{Y} \backslash \mathbb{X}$.

Proof. We have $f^{*} X=0$ if and only if the first map in the triangle (6.5) is an isomorphism.

It is well known that $f^{*}$ induces an equivalence

$$
\mathbf{D}(\mathrm{Q} \operatorname{coh} \mathbb{Y}) / \mathbf{D}_{\mathbb{Y} \backslash \mathbb{X}}(\mathrm{Q} \operatorname{coh} \mathbb{Y}) \stackrel{\sim}{\longrightarrow} \mathbf{D}(\mathrm{Q} \operatorname{coh} \mathbb{X}),
$$

where $\mathbf{D}_{\mathbb{Y} \backslash \mathbb{X}}($ Qcoh $\mathbb{Y})$ denotes the full subcategory of all complexes in $\mathbf{D}(\mathrm{Qcoh} \mathbb{Y})$ such that the support of the cohomology is contained in $\mathbb{Y} \backslash \mathbb{X}$. We obtain an analogue for $\mathbf{K}(\operatorname{Inj} \mathbb{Y})$ and $\mathbf{S}($ Qcoh $\mathbb{Y})$ if we define

$$
\begin{aligned}
\mathbf{K}_{\mathbb{Y} \backslash \mathbb{X}}(\operatorname{Inj} \mathbb{Y}) & =\{X \in \mathbf{K}(\operatorname{Inj} \mathbb{Y}) \mid \operatorname{Supp} X \subseteq \mathbb{Y} \backslash \mathbb{X}\}, \\
\mathbf{S}_{\mathbb{Y} \backslash \mathbb{X}}(\mathrm{Qcoh} \mathbb{Y}) & =\{X \in \mathbf{S}(\text { Qcoh } \mathbb{Y}) \mid \operatorname{Supp} X \subseteq \mathbb{Y} \backslash \mathbb{X}\}
\end{aligned}
$$

Proposition 6.9. Let $\mathbb{Y}$ be a separated noetherian scheme and $f: \mathbb{X} \rightarrow \mathbb{Y}$ be the inclusion of an open subscheme. Then $f^{*}$ induces equivalences

$$
\begin{gathered}
\mathbf{K}(\operatorname{Inj} \mathbb{Y}) / \mathbf{K}_{\mathbb{Y} \backslash \mathbb{X}}(\operatorname{Inj} \mathbb{Y}) \stackrel{\sim}{\longrightarrow} \mathbf{K}(\operatorname{Inj} \mathbb{X}), \\
\mathbf{S}(Q \operatorname{coh} \mathbb{Y}) / \mathbf{S}_{\mathbb{Y} \backslash \mathbb{X}}(Q \operatorname{coh} \mathbb{Y}) \stackrel{\sim}{\longrightarrow} \mathbf{S}(Q \operatorname{coh} \mathbb{X}) .
\end{gathered}
$$

Proof. We have $f^{*} \circ f_{*} \cong \operatorname{Id}_{\mathrm{Qcoh} \mathbb{X}}$, and this carries over to complexes of injectives. On the other hand, we have for $X$ in $\mathbf{K}(\operatorname{Inj} \mathbb{Y})$ a natural map $X \rightarrow\left(f_{*}^{\circ} f^{*}\right) X$ which induces an isomorphism in $\mathbf{K}(\operatorname{Inj} \mathbb{Y}) / \mathbf{K}_{\mathbb{Y} \backslash \mathbb{X}}(\operatorname{Inj} \mathbb{Y})$, by Lemma 6.8. This gives the first equivalence. The second equivalence follows from the first, because $f^{*}$ and $f_{*}$ restrict to functors between $\mathbf{S}($ Qcoh $\mathbb{Y})$ and $\mathbf{S}($ Qcoh $\mathbb{X})$. This is clear for $f^{*}$ because it is exact. For $f_{*}$ this follows from Theorem 1.5.

Let us give a more elaborate formulation of Proposition 6.9. The functor $\hat{\mathbf{R}} f_{*}=f_{*}: \mathbf{K}(\operatorname{Inj} \mathbb{X}) \rightarrow$ $\mathbf{K}(\operatorname{Inj} \mathbb{Y})$ admits a left and a right adjoint. Therefore $\hat{\mathbf{R}} f_{*}$ induces a recollement

$$
\mathbf{K}(\operatorname{Inj} \mathbb{X}) \rightleftarrows \mathbf{K}(\operatorname{Inj} \mathbb{Y}) \Longleftarrow \mathbf{K}_{\mathbb{Y} \backslash \mathbb{X}}(\operatorname{Inj} \mathbb{Y})
$$

This recollement is compatible with the recollement

$$
\mathbf{S}(\mathrm{Qcoh} \mathbb{Y}) \rightleftarrows \mathbf{K}(\operatorname{Inj} \mathbb{Y}) \rightleftarrows \mathbf{D}(\mathrm{Q} \operatorname{coh} \mathbb{Y})
$$

and we obtain the following diagram.

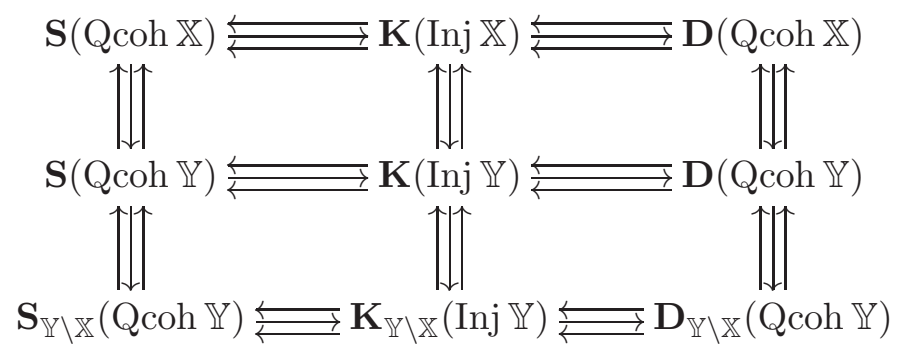

In this diagram, each row and each column is a recollement. Moreover, the diagram is commutative if one restricts to arrows in the south and east directions. All other commutativity relations follow by taking left adjoints or right adjoints.

Proposition 6.9 tells us precisely when the inclusion of a subscheme induces an equivalence for the stable derived category. In [Or103], Orlov observed that the bounded stable derived category of a noetherian scheme depends only on the singular points. We extend this result to the unbounded stable derived category, using a completely different proof. 


\section{H. KRAUSE}

Corollary 6.10. Let $\mathbb{Y}$ be a separated noetherian scheme of finite Krull dimension. If $f: \mathbb{X} \rightarrow \mathbb{Y}$ denotes the inclusion of an open subscheme which contains all singular points of $\mathbb{Y}$, then $\mathbf{S} f^{*}: \mathbf{S}(\mathrm{Qcoh} \mathbb{Y}) \rightarrow \mathbf{S}($ Qcoh $\mathbb{X})$ is an equivalence.

Proof. We apply Proposition 6.9 and need to show that $\mathbf{S}_{\mathbb{Y} \backslash \mathbb{X}}(\mathrm{Qcoh} \mathbb{Y})=0$. However, this is clear from our assumptions on $\mathbb{X}$ and $\mathbb{Y}$.

Orlov's result [Orl03, Proposition 1.14] is an immediate consequence if one restricts the equivalence $\mathbf{S} f^{*}$ to compact objects; see Theorem 6.6.

Corollary 6.11. Let $\mathbb{Y}$ be a separated noetherian scheme of finite Krull dimension. If $f: \mathbb{X} \rightarrow \mathbb{Y}$ denotes the inclusion of an open subscheme which contains all singular points of $\mathbb{Y}$, then $f^{*}$ induces (up to direct factors) an equivalence

$$
\mathbf{D}^{\mathrm{b}}(\operatorname{coh} \mathbb{Y}) / \mathbf{D}^{\text {perf }}(\operatorname{coh} \mathbb{Y}) \longrightarrow \mathbf{D}^{\mathrm{b}}(\operatorname{coh} \mathbb{X}) / \mathbf{D}^{\text {perf }}(\operatorname{coh} \mathbb{X})
$$

\section{Gorenstein injective approximations and Tate cohomology}

Let $\mathcal{A}$ be a locally noetherian Grothendieck category and suppose that the derived category $\mathbf{D}(\mathcal{A})$ is compactly generated. In this section, we study the category of complete injective resolutions. We assign functorially to each complex of injectives a complete resolution. This yields Gorenstein injective approximations and Tate cohomology groups for objects in $\mathcal{A}$. The classical definition of Tate cohomology is based on complete projective resolutions. Our approach is essentially the same, however using resolutions with injective instead of projective components. Another aspect in this section is the interplay between the stable derived category $\mathbf{S}(\mathcal{A})$ and the stable category $\underline{\mathcal{A}}$ modulo injective objects, which is obtained from $\mathcal{A}$ by identifying two maps if their difference factors through some injective object. Given objects $A, B$ in $\mathcal{A}$, we write

$$
\underline{\operatorname{Hom}}_{\mathcal{A}}(A, B)=\operatorname{Hom}_{\underline{\mathcal{A}}}(A, B) .
$$

The functor

$$
\mathbf{K}(\operatorname{Inj} \mathcal{A}) \longrightarrow \underline{\mathcal{A}}, \quad X \mapsto Z^{0} X=\operatorname{Ker}\left(X^{0} \rightarrow X^{1}\right)
$$

provides a link between the stable categories $\mathbf{S}(\mathcal{A})$ and $\underline{\mathcal{A}}$. In particular, we obtain an explicit description of the stabilization functor

$$
S: \mathcal{A} \stackrel{\operatorname{can}}{\longrightarrow} \mathbf{D}(\mathcal{A}) \stackrel{I_{\lambda} \circ Q_{\rho}}{\longrightarrow} \mathbf{S}(\mathcal{A})
$$

provided that $\mathcal{A}$ has some appropriate Gorenstein property.

Most of the concepts in this section are classical, but seem to be new in this setting and this generality. We refer to the end of this section for historical remarks and references to the literature.

Let us start with the relevant definitions. A complex $X$ in $\operatorname{Inj} \mathcal{A}$ is called totally acyclic if $\operatorname{Hom}_{\mathcal{A}}(A, X)$ and $\operatorname{Hom}_{\mathcal{A}}(X, A)$ are acyclic complexes of abelian groups for all $A \operatorname{in} \operatorname{Inj} \mathcal{A}$. We denote by $\mathbf{K}_{\mathrm{tac}}(\operatorname{Inj} \mathcal{A})$ the full subcategory of all totally acyclic complexes in $\mathbf{K}(\operatorname{Inj} \mathcal{A})$. Following [EJ95], we call an object $A$ in $\mathcal{A}$ Gorenstein injective if it is of the form $Z^{0} X$ for some $X$ in $\mathbf{K}_{\mathrm{tac}}(\operatorname{Inj} \mathcal{A})$. We write GInj $\mathcal{A}$ for the full subcategory formed by all Gorenstein injective objects.

Lemma 7.1. Let $\mathcal{A}$ be an abelian category and let $X, Y$ be objects in $\mathbf{K}(\operatorname{Inj} \mathcal{A})$. Suppose that $H^{n} X=0$ for all $n>0$ and $Y$ is totally acyclic. Then the canonical map

$$
\sigma_{X, Y}: \operatorname{Hom}_{\mathbf{K}(\operatorname{Inj} \mathcal{A})}(X, Y) \longrightarrow \underline{\operatorname{Hom}}_{\mathcal{A}}\left(Z^{0} X, Z^{0} Y\right)
$$

is bijective. 


\section{THE STABLE DERIVED CATEGORY OF A NOETHERIAN SCHEME}

Proof. Fix a map $\alpha: Z^{0} X \rightarrow Z^{0} Y$ in $\mathcal{A}$. We need to extend $\alpha$ to a chain map $\bar{\alpha}: X \rightarrow Y$ such that $Z^{0} \bar{\alpha}=\alpha$. We use the assumption on $X$ to extend $\alpha$ in non-negative degrees, and the assumption on $Y$ allows to extend $\alpha$ in negative degrees. Thus, $\sigma_{X, Y}$ is surjective. To show that $\sigma_{X, Y}$ is injective, let $\phi: X \rightarrow Y$ be a chain map such that $Z^{0} \phi$ factors through some injective object. A similar argument as before yields a chain homotopy $X \rightarrow Y$ which shows that $\phi$ is null homotopic. Thus, the proof is complete.

Let us denote by GInj $\underline{\mathcal{A}}$ the full subcategory of $\underline{\mathcal{A}}$ formed by the objects in GInj $\mathcal{A}$. Observe that GInj $\mathcal{A}$ is a Frobenius category with respect to the class of exact sequences from $\mathcal{A}$. With respect to this exact structure, an object $A$ in GInj $\mathcal{A}$ is projective if and only if $A$ is injective if and only if $A$ belongs to $\operatorname{Inj} \mathcal{A}$. Thus, the category $\operatorname{GInj} \underline{\mathcal{A}}$ carries a triangulated structure. The shift takes an object $A$ to the cokernel $\Sigma A$ of a monomorphism $A \rightarrow E$ into an injective object $E$. The exact triangles are induced from short exact sequences in $\mathcal{A}$.

Proposition 7.2. Let $\mathcal{A}$ be an abelian category. Then the functor

$$
\mathbf{K}_{\mathrm{tac}}(\operatorname{Inj} \mathcal{A}) \longrightarrow \operatorname{GInj} \underline{\mathcal{A}}, \quad X \mapsto Z^{0} X,
$$

is an equivalence of triangulated categories.

Proof. We need to show that the functor is fully faithful and surjective on isomorphism classes of objects. The last property is clear from the definition of GInj $\mathcal{A}$. The functor is fully faithful by Lemma 7.1. Finally, observe that an exact triangle of complexes comes, up to isomorphism, from a sequence of complexes which is split exact in each degree. Thus, we obtain an exact sequence in $\mathcal{A}$ and an exact triangle in $\underline{\mathcal{A}}$ if we apply $Z^{0}$.

The following lemma is crucial because it provides the existence of complete injective resolutions. Let us write

$$
G: \mathbf{K}_{\mathrm{tac}}(\operatorname{Inj} \mathcal{A}) \longrightarrow \mathbf{K}(\operatorname{Inj} \mathcal{A})
$$

for the inclusion functor.

Lemma 7.3. Let $\mathcal{A}$ be a locally noetherian Grothendieck category and suppose that $\mathbf{D}(\mathcal{A})$ is compactly generated. Then the inclusion $G: \mathbf{K}_{\operatorname{tac}}(\operatorname{Inj} \mathcal{A}) \rightarrow \mathbf{K}(\operatorname{Inj} \mathcal{A})$ has a left adjoint

$$
G_{\lambda}: \mathbf{K}(\operatorname{Inj} \mathcal{A}) \longrightarrow \mathbf{K}_{\operatorname{tac}}(\operatorname{Inj} \mathcal{A}) \text {. }
$$

Proof. The inclusion $I: \mathbf{K}_{\mathrm{ac}}(\operatorname{Inj} \mathcal{A}) \rightarrow \mathbf{K}(\operatorname{Inj} \mathcal{A})$ has a left adjoint $I_{\lambda}$ by Theorem 4.2. Thus, it is sufficient to show that the inclusion $\mathbf{K}_{\mathrm{tac}}(\operatorname{Inj} \mathcal{A}) \rightarrow \mathbf{K}_{\mathrm{ac}}(\operatorname{Inj} \mathcal{A})$ has a left adjoint. Let us denote by $E$ the coproduct of a representative set of all indecomposable injective objects in $\mathcal{A}$. By definition, $\mathbf{K}_{\mathrm{tac}}(\operatorname{Inj} \mathcal{A})$ consists of all objects $X$ in $\mathbf{K}_{\mathrm{ac}}(\operatorname{Inj} \mathcal{A})$ such that

$$
\operatorname{Hom}_{\mathbf{K}(\operatorname{Inj} \mathcal{A})}\left(\Sigma^{n} E, X\right) \cong \operatorname{Hom}_{\mathbf{K}_{\mathrm{ac}}(\operatorname{Inj} \mathcal{A})}\left(I_{\lambda}\left(\Sigma^{n} E\right), X\right)
$$

vanishes for all $n \in \mathbb{Z}$. The category $\mathbf{K}_{\mathrm{ac}}(\operatorname{Inj} \mathcal{A})$ is compactly generated by Corollary 5.4 , and we can apply Proposition 3.4 to obtain a left adjoint for the inclusion $\mathbf{K}_{\text {tac }}(\operatorname{Inj} \mathcal{A}) \rightarrow \mathbf{K}_{\mathrm{ac}}(\operatorname{Inj} \mathcal{A})$.

Given an object $A$ in $\mathcal{A}$ with injective resolution $i A$, we call the natural map

$$
i A \longrightarrow G_{\lambda} i A
$$

a complete injective resolution of $A$. If we apply the functor $Z^{0}$ to this map, we obtain a Gorenstein injective approximation of $A$.

Theorem 7.4. Let $\mathcal{A}$ be a locally noetherian Grothendieck category and suppose that $\mathbf{D}(\mathcal{A})$ is compactly generated. Then the inclusion $\operatorname{GInj} \underline{\mathcal{A}} \rightarrow \underline{\mathcal{A}}$ has a left adjoint

$$
T: \underline{\mathcal{A}} \longrightarrow \operatorname{GInj} \underline{\mathcal{A}} \text {. }
$$




\section{H. KRAUSE}

Thus, for each object $A$ in $\mathcal{A}$ we have a natural map $A \rightarrow T A$ which induces a bijection

$$
\underline{\operatorname{Hom}}_{\mathcal{A}}(T A, B) \stackrel{\sim}{\longrightarrow} \underline{\operatorname{Hom}}_{\mathcal{A}}(A, B) \quad \text { for all } B \in \operatorname{GInj} \mathcal{A} \text {. }
$$

Proof. Fix an object $A$ in $\mathcal{A}$ and choose an injective resolution $i A$. We put

$$
T A=Z^{0}\left(G_{\lambda} i A\right)
$$

and this induces a functor $T: \underline{\mathcal{A}} \rightarrow \operatorname{GInj} \underline{\mathcal{A}}$. Let $B$ in GInj $\mathcal{A}$ and fix a totally acyclic complex $t B$ such that $Z^{0} t B=B$. The natural map $i A \rightarrow G_{\lambda} i A$ induces a map $A \rightarrow T A$ in $\underline{\mathcal{A}}$ which makes the following square commutative.

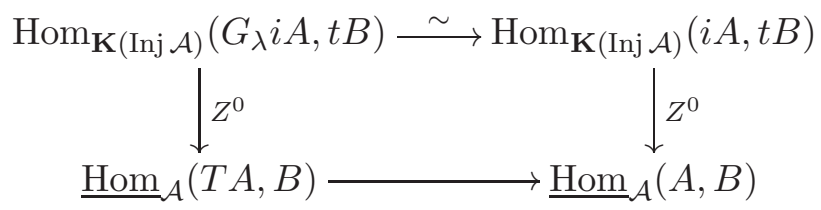

The vertical maps are bijective by Lemma 7.1, and we conclude that $T$ is a left adjoint for the inclusion GInj $\underline{\mathcal{A}} \rightarrow \underline{\mathcal{A}}$.

Next we use complete injective resolutions to define Tate cohomology groups for objects in $\mathcal{A}$.

Definition 7.5. Given objects $A, B$ in $\mathcal{A}$ and $n \in \mathbb{Z}$, the Tate cohomology group is

$$
\widehat{\operatorname{Ext}}_{\mathcal{A}}^{n}(A, B)=H^{n} \operatorname{Hom}_{\mathcal{A}}\left(A, G_{\lambda} i B\right)
$$

Remark 7.6. The correct term for this cohomology theory would be 'injective Tate cohomology' in order to distinguish it from the usual 'projective Tate cohomology', which is defined via complete projective resolutions. For simplicity, we drop the extra adjective 'injective'. Note that confusion is not possible because we do not consider projective Tate cohomology in this paper.

Tate cohomology is natural in both arguments because the formation of complete injective resolutions is functorial. In addition, we have a comparison map

$$
\operatorname{Ext}_{\mathcal{A}}^{n}(A, B) \longrightarrow \widehat{\operatorname{Ext}}_{\mathcal{A}}^{n}(A, B),
$$

which is induced by the map $i B \rightarrow G_{\lambda} i B$. There is an alternative description of Tate cohomology which is based on the left adjoint $T: \underline{\mathcal{A}} \rightarrow \operatorname{GInj} \underline{\mathcal{A}}$.

Proposition 7.7. Given objects $A, B$ in $\mathcal{A}$ and $n \in \mathbb{Z}$, there is a natural isomorphism

$$
\widehat{\operatorname{Ext}}_{\mathcal{A}}^{n}(A, B) \cong \underline{\operatorname{Hom}}_{\mathcal{A}}\left(A, \Sigma^{n}(T B)\right) .
$$

Proof. Using Lemmas 2.1 and 7.1, we have the following sequence of isomorphisms

$$
\begin{aligned}
H^{n} \operatorname{Hom}_{\mathcal{A}}\left(A, G_{\lambda} i B\right) & \cong \operatorname{Hom}_{\mathbf{K}(\mathcal{A})}\left(A, \Sigma^{n}\left(G_{\lambda} i B\right)\right) \\
& \cong \operatorname{Hom}_{\mathbf{K}(\mathcal{A})}\left(i A, \Sigma^{n}\left(G_{\lambda} i B\right)\right) \\
& \cong \underline{\operatorname{Hom}}_{\mathcal{A}}\left(A, \Sigma^{n}(T B)\right) .
\end{aligned}
$$

We have a more conceptual definition of Tate cohomology for objects in $\mathbf{D}(\mathcal{A})$ which uses the composite

$$
U: \mathbf{D}(\mathcal{A}) \stackrel{G_{\lambda} \circ Q_{\rho}}{\longrightarrow} \mathbf{K}_{\mathrm{tac}}(\operatorname{Inj} \mathcal{A}) .
$$

Thus, for objects $X, Y$ in $\mathbf{D}(\mathcal{A})$ and $n \in \mathbb{Z}$ we define

$$
\widehat{\operatorname{Ext}}_{\mathcal{A}}^{n}(X, Y)=\operatorname{Hom}_{\mathbf{K}(\mathcal{A})}\left(U X, \Sigma^{n}(U Y)\right) \text {. }
$$

Note that this definition is consistent with the original definition of Tate cohomology if we take objects in $\mathcal{A}$ and view them as complexes concentrated in degree 0 . This follows from the fact 


\section{THE STABLE DERIVED CATEGORY OF A NOETHERIAN SCHEME}

that $Q_{\rho} X$ is simply an injective resolution of $X^{0}$ when $X$ is concentrated in degree 0 . From now on, we use one of the alternative descriptions of Tate cohomology whenever this is convenient.

Next we show that each exact sequence in $\mathcal{A}$ induces a long exact sequence in Tate cohomology. This is based on the following simple lemma.

Lemma 7.8. The left adjoint $T: \underline{\mathcal{A}} \rightarrow \operatorname{GInj} \underline{\mathcal{A}}$ has the following properties.

(1) An exact sequence $0 \rightarrow A^{\prime} \rightarrow A \rightarrow A^{\prime \prime} \rightarrow 0$ in $\mathcal{A}$ induces an exact triangle

$$
T A^{\prime} \longrightarrow T A \longrightarrow T A^{\prime \prime} \longrightarrow \Sigma\left(T A^{\prime}\right) \quad \text { in } \operatorname{GInj} \underline{\mathcal{A}} \text {. }
$$

(2) Let $A, B$ be in $\mathcal{A}$ and $n \in \mathbb{Z}$. The natural map $A \rightarrow T A$ induces an isomorphism

$$
\widehat{\operatorname{Ext}}_{\mathcal{A}}^{n}(T A, B) \cong \widehat{\operatorname{Ext}}_{\mathcal{A}}^{n}(A, B) \text {. }
$$

Proof. (1) We have an exact triangle $A^{\prime} \rightarrow A \rightarrow A^{\prime \prime} \rightarrow \Sigma\left(A^{\prime}\right)$ in $\mathbf{D}(\mathcal{A})$. Now use that the exact functor $Z^{0} \circ G_{\lambda} \circ Q_{\rho}$ computes $T$.

(2) The adjointness property of $T$ implies $\underline{\operatorname{Hom}}_{\mathcal{A}}(T A, T B) \cong \underline{\operatorname{Hom}}_{\mathcal{A}}(A, T B)$.

Proposition 7.9. Let $0 \rightarrow B^{\prime} \rightarrow B \rightarrow B^{\prime \prime} \rightarrow 0$ be an exact sequence in $\mathcal{A}$. Then we have for $A$ and $C$ in $\mathcal{A}$ the following long exact sequences

$$
\begin{aligned}
\cdots \longrightarrow \widehat{\operatorname{Ext}}_{\mathcal{A}}^{n}\left(A, B^{\prime}\right) \longrightarrow \widehat{\operatorname{Ext}}_{\mathcal{A}}^{n}(A, B) \longrightarrow \widehat{\operatorname{Ext}}_{\mathcal{A}}^{n}\left(A, B^{\prime \prime}\right) \\
\longrightarrow \widehat{\operatorname{Ext}}_{\mathcal{A}}^{n+1}\left(A, B^{\prime}\right) \longrightarrow \widehat{\operatorname{Ext}}_{\mathcal{A}}^{n+1}(A, B) \longrightarrow \widehat{\operatorname{Ext}}_{\mathcal{A}}^{n+1}\left(A, B^{\prime \prime}\right) \longrightarrow \cdots \\
\cdots \widehat{\operatorname{Ext}}_{\mathcal{A}}^{n}\left(B^{\prime \prime}, C\right) \longrightarrow \widehat{\operatorname{Ext}}_{\mathcal{A}}^{n}(B, C) \longrightarrow \widehat{\operatorname{Ext}}_{\mathcal{A}}^{n}\left(B^{\prime}, C\right) \\
\quad \longrightarrow \widehat{\operatorname{Ext}}_{\mathcal{A}}^{n+1}\left(B^{\prime \prime}, C\right) \longrightarrow \widehat{\operatorname{Ext}}_{\mathcal{A}}^{n+1}(B, C) \longrightarrow \widehat{\operatorname{Ext}}_{\mathcal{A}}^{n+1}\left(B^{\prime}, C\right) \longrightarrow \cdots
\end{aligned}
$$

Proof. We apply Lemma 7.8 and use the fact that $\underline{\operatorname{Hom}}_{\mathcal{A}}(T A,-)$ and $\underline{\operatorname{Hom}}_{\mathcal{A}}(-, T C)$ are cohomological functors.

We compute Tate cohomology for Gorenstein injective objects.

Proposition 7.10. Let $A, B$ be objects in $\mathcal{A}$ and suppose that $B$ is Gorenstein injective. Then the comparison map

$$
\operatorname{Ext}_{\mathcal{A}}^{n}(A, B) \longrightarrow \widehat{\operatorname{Ext}}_{\mathcal{A}}^{n}(A, B)
$$

is an isomorphism for $n>0$ and induces an isomorphism

$$
\underline{\operatorname{Hom}}_{\mathcal{A}}(A, B) \stackrel{\sim}{\longrightarrow} \widehat{\operatorname{Ext}}_{\mathcal{A}}^{0}(A, B) \text { for } n=0 .
$$

Proof. Our assumption implies $T B=B$. The case $n=0$ is clear. For $n=1$, choose an exact sequence $0 \rightarrow B \rightarrow E \rightarrow \Sigma B \rightarrow 0$ with $E$ injective and apply $\operatorname{Hom}_{\mathcal{A}}(A,-)$. The cokernel of

$$
\operatorname{Hom}_{\mathcal{A}}(A, E) \longrightarrow \operatorname{Hom}_{\mathcal{A}}(A, \Sigma B)
$$

is isomorphic $\operatorname{Ext}_{\mathcal{A}}^{1}(A, B)$; it is isomorphic to $\underline{\operatorname{Hom}}_{\mathcal{A}}(A, \Sigma B)$ since $B$ is Gorenstein injective. For $n>1$, use dimension shift.

Next we describe those objects $A$ in $\mathcal{A}$ such that $\widehat{\operatorname{Ext}}_{\mathcal{A}}^{*}(A,-)$ vanishes. For instance, Tate cohomology vanishes for all objects having finite projective or finite injective dimension.

Proposition 7.11. For an object $A$ in $\mathcal{A}$, the following are equivalent.

(1) $\widehat{\operatorname{Ext}}_{\mathcal{A}}^{*}(A,-)=0$.

(2) $\widehat{\operatorname{Ext}}_{\mathcal{A}}^{0}(A, A)=0$. 


\section{H. KRAUSE}

(3) $\widehat{\operatorname{Ext}}_{\mathcal{A}}^{*}(-, A)=0$.

(4) $\operatorname{Ext}_{\mathcal{A}}^{1}(A, B)=0$ for all $B \in \operatorname{GInj} \mathcal{A}$.

(5) $\underline{\operatorname{Hom}}_{\mathcal{A}}(A, B)=0$ for all $B \in \operatorname{GInj} \mathcal{A}$.

Proof. Use the isomorphism $\widehat{\operatorname{Ext}}_{\mathcal{A}}^{*}(-,-) \cong \underline{\operatorname{Hom}}_{\mathcal{A}}^{*}(T-, T-)$ and the fact that $\widehat{\operatorname{Ext}}_{\mathcal{A}}^{1}(-, B)$ can be computed via $\operatorname{Ext}_{\mathcal{A}}^{1}(-, T B)$.

The following result formulates our analogue of the maximal Cohen-Macaulay approximation in the sense of Auslander and Buchweitz [AB89]. Note that a Gorenstein injective object is the 'dual' of a maximal Cohen-Macaulay object which one defines in a category with enough projectives. Let

$$
\mathcal{X}=\left\{A \in \mathcal{A} \mid \operatorname{Ext}_{\mathcal{A}}^{1}(A, B)=0 \text { for all } B \in \operatorname{GInj} \mathcal{A}\right\} \quad \text { and } \quad \mathcal{Y}=\operatorname{GInj} \mathcal{A} .
$$

Theorem 7.12. Let $\mathcal{A}$ be a locally noetherian Grothendieck category and suppose that $\mathbf{D}(\mathcal{A})$ is compactly generated.

(1) Every object $A$ in $\mathcal{A}$ fits into exact sequences

$$
0 \rightarrow Y_{A} \rightarrow X_{A} \rightarrow A \rightarrow 0 \text { and } 0 \rightarrow A \rightarrow Y^{A} \rightarrow X^{A} \rightarrow 0
$$

in $\mathcal{A}$ with $X_{A}, X^{A}$ in $\mathcal{X}$ and $Y_{A}, Y^{A}$ in $\mathcal{Y}$.

(2) The map $A \mapsto X_{A}$ induces a right adjoint for the inclusion $\underline{\mathcal{X}} \rightarrow \underline{\mathcal{A}}$.

(3) The map $A \mapsto Y^{A}$ induces a left adjoint for the inclusion $\underline{\mathcal{Y}} \rightarrow \underline{\mathcal{A}}$.

(4) $\mathcal{X} \cap \mathcal{Y}=\operatorname{Inj} \mathcal{A}$.

Note that this is essentially the statement of Theorem 1.3 from the introduction, since $\mathcal{X}$ is precisely the subcategory of objects $A$ in $\mathcal{A}$ such that the Tate cohomology functor $\widehat{\operatorname{Ext}}_{\mathcal{A}}^{*}(A,-)$ vanishes.

Proof. We use the basic properties of Tate cohomology.

(1) Fix an object $A$ in $\mathcal{A}$ and a complete injective resolution

$$
i A \longrightarrow G_{\lambda} i A=y A .
$$

We complete this map to an exact triangle

$$
i A \longrightarrow y A \longrightarrow x A \longrightarrow \Sigma(i A) .
$$

in $\mathbf{K}(\operatorname{Inj} \mathcal{A})$ and therefore have a sequence $0 \rightarrow i A \rightarrow y A \rightarrow x A \rightarrow 0$ of complexes which is split exact in each degree. Applying $Z^{0}: \mathbf{K}(\operatorname{Inj} \mathcal{A}) \rightarrow \underline{\mathcal{A}}$ produces an exact sequence

$$
0 \longrightarrow A \stackrel{\alpha}{\longrightarrow} Y^{A} \stackrel{\beta}{\longrightarrow} X^{A} \longrightarrow 0
$$

in $\mathcal{A}$. Clearly, $Y^{A}$ belongs to $\mathcal{Y}$. On the other hand, $\widehat{\operatorname{Ext}}_{\mathcal{A}}^{*}(\alpha,-)$ is an isomorphism. Thus, $\widehat{\operatorname{Ext}}_{\mathcal{A}}^{*}\left(X^{A},-\right)$ vanishes and $X^{A}$ belongs to $\mathcal{X}$. The second sequence ending in $A$ is obtained by rotating the triangle (7.1).

(2) We consider the exact sequence

$$
0 \longrightarrow Y_{A} \stackrel{\mu}{\longrightarrow} X_{A} \stackrel{\nu}{\longrightarrow} A \longrightarrow 0
$$

and need to show that $\underline{\operatorname{Hom}}_{\mathcal{A}}(X, \nu)$ is bijective for all $X$ in $\mathcal{X}$. To see this, let $\phi: X \rightarrow A$ be a map with $X$ in $\mathcal{X}$. The map $\phi$ factors through $\nu$ since $\operatorname{Ext}_{\mathcal{A}}^{1}\left(X, Y_{A}\right)=0$. Therefore, $\underline{\operatorname{Hom}}_{\mathcal{A}}(X, \nu)$ is surjective. To show that $\underline{\operatorname{Hom}}_{\mathcal{A}}(X, \nu)$ is injective, let $\psi: X \rightarrow X_{A}$ be a map such that $\nu \circ \psi$ has a factorization $X \stackrel{\phi^{\prime}}{\rightarrow} E \stackrel{\phi^{\prime \prime}}{\rightarrow} A$ with injective $E$. We obtain a factorization $\phi^{\prime \prime}=\nu \circ \chi$, since $E$ belongs to $\mathcal{X}$. We have $\nu \circ\left(\psi-\chi \circ \phi^{\prime}\right)=0$, and $\psi-\chi \circ \phi^{\prime}$ needs to factor through $\mu$. Therefore, $\psi-\chi \circ \phi^{\prime}$ 
factors through some injective object, since $\underline{\operatorname{Hom}}_{\mathcal{A}}\left(X, Y_{A}\right)=0$. We conclude that $\psi$ factors through an injective object. Thus, the map $\underline{\operatorname{Hom}}_{\mathcal{A}}(X, \nu)$ is bijective.

(3) We consider the exact sequence

$$
0 \longrightarrow A \stackrel{\alpha}{\longrightarrow} Y^{A} \stackrel{\beta}{\longrightarrow} X^{A} \longrightarrow 0
$$

and need to show that $\underline{\operatorname{Hom}}_{\mathcal{A}}(\alpha, Y)$ is bijective for all $Y$ in $\mathcal{Y}$. However, this is clear from the long exact sequence for Tate cohomology, since $\underline{\operatorname{Hom}}_{\mathcal{A}}(-, Y) \cong \widehat{\operatorname{Ext}}_{\mathcal{A}}^{0}(-, Y)$ and $\widehat{\operatorname{Ext}}_{\mathcal{A}}^{*}\left(X^{A},-\right)$ vanishes.

(4) Clearly, Inj $\mathcal{A}$ is contained in $\mathcal{X} \cap \mathcal{Y}$. Now let $A$ be in $\mathcal{X} \cap \mathcal{Y}$. Thus,

$$
\underline{\operatorname{Hom}}_{\mathcal{A}}(A, A) \cong \widehat{\operatorname{Ext}}_{\mathcal{A}}^{0}(A, A)=0 .
$$

Therefore, the identity map $A \rightarrow A$ factors through an injective object. We conclude that $A$ is injective.

Let us comment on the interplay between the stable category $\underline{\mathcal{A}}$ and the stable derived category $\mathbf{S}(\mathcal{A})$. We have already seen that the definition of Tate cohomology is possible in both settings. It is more elementary in $\underline{\mathcal{A}}$, but more conceptual using the category of complete injective resolutions $\mathbf{K}_{\text {tac }}(\operatorname{Inj} \mathcal{A})$ which is a subcategory of $\mathbf{S}(\mathcal{A})$. The same phenomenon appears when one studies Gorenstein injective approximations. The proof of Theorem 7.12 we have given uses the category of complexes $\mathbf{K}(\operatorname{Inj} \mathcal{A})$. There is an alternative proof which avoids complexes and instead uses the left adjoint $T: \underline{\mathcal{A}} \rightarrow \operatorname{GInj} \underline{\mathcal{A}}$.

Gorenstein rings and schemes play an important role in applications and have a number of interesting homological properties. It is therefore important to formulate a Gorenstein property for a locally noetherian Grothendieck category $\mathcal{A}$. Let us denote by $\Sigma^{\infty} \mathcal{A}$ the full subcategory of objects $A$ in $\mathcal{A}$ which fit into an exact sequence

$$
\cdots \longrightarrow E_{2} \longrightarrow E_{1} \longrightarrow E_{0} \longrightarrow A \longrightarrow 0
$$

with $E_{n}$ injective for all $n$. We say that $\mathcal{A}$ has the injective Gorenstein property if the equivalent conditions in the following proposition are satisfied. This property has been studied by Beligiannis in $[$ Bel00].

Proposition 7.13. Let $\mathcal{A}$ be a locally noetherian Grothendieck category and suppose that $\mathbf{D}(\mathcal{A})$ is compactly generated. Then the following are equivalent.

(1) $\operatorname{Ext}_{\mathcal{A}}^{1}(A, B)=0$ for all $A \in \operatorname{Inj} \mathcal{A}$ and $B \in \Sigma^{\infty} \mathcal{A}$.

(2) Every acyclic complex in $\operatorname{Inj} \mathcal{A}$ is totally acyclic.

(3) $\operatorname{GInj} \mathcal{A}=\Sigma^{\infty} \mathcal{A}$.

(4) $S: \mathcal{A} \stackrel{\text { can }}{\longrightarrow} \mathbf{D}(\mathcal{A}) \stackrel{I_{\lambda}{ }^{\circ} Q_{\rho}}{\longrightarrow} \mathbf{S}(\mathcal{A})$ annihilates all injective objects.

(5) $S$ induces an equivalence $\operatorname{GInj} \underline{\mathcal{A}} \rightarrow \mathbf{S}(\mathcal{A})$.

Proof. Conditions (1)-(3) are pairwise equivalent. This follows from the formula

$$
\operatorname{Ext}_{\mathcal{A}}^{n}\left(A, Z^{0} Y\right) \cong \operatorname{Hom}_{\mathbf{K}(\mathcal{A})}\left(A, \Sigma^{n} Y\right) \cong H^{n} \operatorname{Hom}_{\mathcal{A}}(A, Y)
$$

where $A$ is any object in $\mathcal{A}$ and $Y$ is an acyclic complex in $\operatorname{Inj} \mathcal{A}$. The first isomorphism is valid for all $n \geqslant 1$, and the second for all $n \in \mathbb{Z}$.

Now observe that $I_{\lambda}$ annihilates precisely those objects $X$ in $\mathbf{K}(\operatorname{Inj} \mathcal{A})$ such that

$$
\operatorname{Hom}_{\mathbf{K}(\operatorname{Inj} \mathcal{A})}(X, Y)=0
$$

for every acyclic complex $Y$ in $\operatorname{Inj} \mathcal{A}$. On the other hand, $\mathcal{A} \rightarrow \mathbf{D}(\mathcal{A})$ and $Q_{\rho}$ are faithful. Thus (1)-(3) are equivalent to (4). Also, (5) implies (4). So, it remains to show that (1)-(4) imply (5). 


\section{H. KRAUSE}

Suppose (2) and (4) hold. We have already seen in Proposition 7.2 that

$$
\mathbf{S}(\mathcal{A}) \longrightarrow \operatorname{GInj} \underline{\mathcal{A}}, \quad X \mapsto Z^{0} X,
$$

is an equivalence, since every acyclic complex is totally acyclic. On the other hand, $S$ annihilates all injective objects and therefore induces a functor $\underline{\mathcal{A}} \rightarrow \mathbf{S}(\mathcal{A})$. The composite with $Z^{0}: \mathbf{S}(\mathcal{A}) \rightarrow \operatorname{GInj} \underline{\mathcal{A}}$ is precisely the right adjoint of the inclusion $\operatorname{GInj} \underline{\mathcal{A}} \rightarrow \underline{\mathcal{A}}$ constructed in Theorem 7.4. Thus, $\left(Z^{0} \circ S\right) A \cong A$ for all $A$ in $\operatorname{GInj} \mathcal{A}$.

Corollary 7.14. Let $\mathcal{A}$ be a locally noetherian Grothendieck category and suppose that $\mathbf{D}(\mathcal{A})$ is compactly generated. If $\mathcal{A}$ has the injective Gorenstein property, then the composite

$$
\operatorname{GInj} \mathcal{A} \stackrel{\text { inc }}{\longrightarrow} \mathcal{A} \stackrel{\text { can }}{\longrightarrow} \mathbf{D}(\mathcal{A}) \stackrel{I_{\lambda} \circ Q_{\rho}}{\longrightarrow} \mathbf{S}(\mathcal{A}) \stackrel{Z^{0}}{\longrightarrow} \operatorname{GInj} \underline{\mathcal{A}}
$$

is naturally isomorphic to the canonical projection $\operatorname{GInj} \mathcal{A} \rightarrow \operatorname{GInj} \underline{\mathcal{A}}$.

We are now in a position to describe the stabilization functor $S: \mathcal{A} \rightarrow \mathbf{S}(\mathcal{A})$, provided that $\mathcal{A}$ has the injective Gorenstein property. We use the left adjoint $T: \underline{\mathcal{A}} \rightarrow \operatorname{GInj} \underline{\mathcal{A}}$ of the inclusion $\operatorname{GInj} \underline{\mathcal{A}} \rightarrow \underline{\mathcal{A}}$. For $A$ in $\mathcal{A}$, choose any acyclic complex $X$ of injective objects such that $Z^{0} X \cong T A$. Then $S A \cong X$.

Example 7.15. Let $\Lambda$ be a ring and suppose $\Lambda$ is Gorenstein; that is, $\Lambda$ is two-sided noetherian and $\Lambda$ has finite injective dimension as left and right $\Lambda$-module. In this case, the category $\operatorname{Mod} \Lambda$ has the injective Gorenstein property. This follows from the fact that every injective $\Lambda$-module has finite projective dimension if $\Lambda$ is Gorenstein; see Example 5.6. Given a $\Lambda$-module $A$, Tate cohomology $\widehat{\operatorname{Ext}}_{\Lambda}^{*}(A,-)$ vanishes if and only if $A$ has finite injective dimension if and only if $A$ has finite projective dimension. Note that for Gorenstein rings, the classical Tate cohomology defined via complete projective resolutions coincides with our Tate cohomology, which is defined via complete injective resolutions; see [BR02].

Example 7.16. Let $\Lambda$ be a ring and suppose that projective and injective $\Lambda$-modules coincide. Then every $\Lambda$-module is Gorenstein injective. In particular, $\mathbf{S}(\operatorname{Mod} \Lambda)$ is equivalent to the stable category $\underline{\mathcal{A}}$ of $\mathcal{A}=\operatorname{Mod} \Lambda$. Given a $\Lambda$-module $A$, there is an exact triangle

$$
p A \longrightarrow i A \longrightarrow t A \longrightarrow \Sigma(p A)
$$

in $\mathbf{K}(\operatorname{Inj} \mathcal{A})$ where $p A$ denotes a projective, $i A$ an injective, and $t A$ a Tate resolution of $A$. This triangle is isomorphic to the canonical triangle

$$
\left(Q_{\lambda} \circ Q\right) \bar{A} \longrightarrow \bar{A} \longrightarrow\left(I \circ I_{\lambda}\right) \bar{A} \longrightarrow \Sigma\left(Q_{\lambda} \circ Q\right) \bar{A}
$$

where $\bar{A}=Q_{\rho} A$.

Example 7.17. Let $\mathbb{X}$ be a noetherian scheme and suppose that every injective object $E$ in $Q \operatorname{coh} \mathbb{X}$ admits a finite resolution

$$
0 \longrightarrow L_{r} \longrightarrow \cdots \longrightarrow L_{2} \longrightarrow L_{1} \longrightarrow L_{0} \longrightarrow E \longrightarrow 0
$$

with $L_{n}$ locally free for each $n$. Then the category Qcoh $\mathbb{X}$ has the injective Gorenstein property.

\section{Historical remarks}

Gorenstein injective approximations and Tate cohomology have a long history. Auslander and Bridger [AB69] introduced the stable module category and assigned to each module a G-dimension. Over Gorenstein rings, the modules of G-dimension 0 are precisely the maximal Cohen-Macaulay or Gorenstein projective modules. Auslander and Buchweitz established maximal Cohen-Macaulay approximations in [AB89], and there is an alternative unpublished approach by Buchweitz [Buc87] 
which involves the derived category. Enochs and his collaborators dropped finiteness conditions on modules and proved the existence of Gorenstein projective and Gorenstein injective approximations for arbitrary modules, for instance over Gorenstein rings [EJ00]. Further generalizations can be found in work of Beligiannis [Bel00]. Jørgensen [Jor01] constructed Gorenstein projective approximations for artin algebras via Bousfield localization, using the category of complete projective resolutions. Hovey [Hov02] and Beligiannis and Reiten [BR02] employed the formalism of model category structures and cotorsion pairs.

The exposition of Buchweitz [Buc87] discussed the close connection between maximal CohenMacaulay approximations and Tate cohomology over Gorenstein rings. For more general settings, we refer to the work of Beligiannis and Reiten [BR02]. A paper of Mislin explained Tate cohomology via satellites [Mis94]. A comparison of Tate cohomology via projectives and injectives was carried out in [Nuc98]. Another exposition of Tate cohomology over noetherian rings can be found in a paper of Avramov and Martsinkovsky [AM02].

\section{Tensor products in modular representation theory}

Let $G$ be a finite group and $k$ be a field. The stable module category $\underline{\operatorname{Mod}} k G$ of the group algebra $k G$ plays an important role in modular representation theory. In this section, we show that this category is equivalent to the stable derived category $\mathbf{S}(\operatorname{Mod} k G)$ and study its tensor product.

It is convenient to work in a slightly more general setting. Thus, we fix a finite-dimensional cocommutative Hopf algebra $\Lambda$ over a field $k$ and consider the module category $\mathcal{A}=\operatorname{Mod} \Lambda$. Note that projective and injective modules over $\Lambda$ coincide. The tensor product $\otimes_{k}$ over $k$ induces a tensor product on $\mathcal{A}$ which extends to a tensor product on $\mathbf{K}(\mathcal{A})$. Similarly, $\operatorname{Hom}_{k}(-,-)$ induces products on $\mathcal{A}$ and $\mathbf{K}(\mathcal{A})$. Note that we have a natural isomorphism

$$
\operatorname{Hom}_{\mathbf{K}(\mathcal{A})}\left(X \otimes_{k} Y, Z\right) \cong \operatorname{Hom}_{\mathbf{K}(\mathcal{A})}\left(X, \operatorname{Hom}_{k}(Y, Z)\right)
$$

for all $X, Y, Z$ in $\mathbf{K}(\mathcal{A})$. The subcategories $\mathbf{K}(\operatorname{Inj} \mathcal{A})$ and $\mathbf{S}(\mathcal{A})$ inherit tensor products from $\mathbf{K}(\mathcal{A})$ because of the following elementary fact.

Lemma 8.1. The subcategories $\mathbf{K}(\operatorname{Inj} \mathcal{A})$ and $\mathbf{S}(\mathcal{A})$ are tensor ideals in $\mathbf{K}(\mathcal{A})$. More precisely:

(1) $X \in \mathbf{K}(\operatorname{Inj} \mathcal{A})$ and $Y \in \mathbf{K}(\mathcal{A})$ imply $X \otimes_{k} Y \in \mathbf{K}(\operatorname{Inj} \mathcal{A})$;

(2) $X \in \mathbf{S}(\mathcal{A})$ and $Y \in \mathbf{K}(\mathcal{A})$ imply $X \otimes_{k} Y \in \mathbf{S}(\mathcal{A})$.

Now consider $k$ as a $\Lambda$-module and view it as a complex concentrated in degree zero; it is the unit of the tensor product in $\mathbf{K}(\mathcal{A})$. This complex fits into exact triangles

$$
a k \longrightarrow k \longrightarrow i k \longrightarrow \Sigma(a k) \text { and } p k \longrightarrow i k \longrightarrow t k \longrightarrow \Sigma(p k)
$$

in $\mathbf{K}(\mathcal{A})$, where $i k$ denotes an injective resolution and $p k$ a projective resolution of $k$ in $\mathcal{A}$. We consider the canonical maps $k \rightarrow i k$ and $p k \rightarrow i k$. Thus, $a k$ and $t k$ are acyclic complexes. In fact, $t k$ is a Tate resolution of $k$ which is obtained by splicing together $p k$ and $i k$.

We have seen in previous sections that the inclusions

$$
\mathbf{S}(\mathcal{A}) \longrightarrow \mathbf{K}(\operatorname{Inj} \mathcal{A}) \quad \text { and } \quad \mathbf{K}(\operatorname{Inj} \mathcal{A}) \longrightarrow \mathbf{K}(\mathcal{A})
$$

have left adjoints. Next we provide explicit descriptions of these adjoints. In particular, we see that they preserve the tensor product $\otimes_{k}$. Note that Hovey et al. pointed out the relevance of these categories in their work on axiomatic stable homotopy theory [HPS97]; we refer to this work for further details and applications.

Theorem 8.2. Let $\Lambda$ be a finite-dimensional cocommutative Hopf algebra over a field $k$, and let $\mathcal{A}=\operatorname{Mod} \Lambda$. 


\section{H. KRAUSE}

(1) The functor

$$
-\otimes_{k} i k: \mathbf{K}(\mathcal{A}) \longrightarrow \mathbf{K}(\operatorname{Inj} \mathcal{A})
$$

is a left adjoint for the inclusion $\mathbf{K}(\operatorname{Inj} \mathcal{A}) \rightarrow \mathbf{K}(\mathcal{A})$.

(2) The functor

$$
-\otimes_{k} t k: \mathbf{K}(\operatorname{Inj} \mathcal{A}) \longrightarrow \mathbf{S}(\mathcal{A})
$$

is a left adjoint for the inclusion $\mathbf{S}(\mathcal{A}) \rightarrow \mathbf{K}(\operatorname{Inj} \mathcal{A})$.

(3) The functor

$$
\mathcal{A} \stackrel{\text { can }}{\longrightarrow} \mathbf{K}(\mathcal{A}) \stackrel{-\otimes t k}{\longrightarrow} \mathbf{S}(\mathcal{A})
$$

induces an equivalence $\underline{\mathcal{A}} \rightarrow \mathbf{S}(\mathcal{A})$ with quasi-inverse $Z^{0}: \mathbf{S}(\mathcal{A}) \rightarrow \underline{\mathcal{A}}$.

Proof. (1) Fix an object $X$ in $\mathbf{K}(\mathcal{A})$. The map $X \cong X \otimes_{k} k \rightarrow X \otimes_{k} i k$ induces for all $Y$ in $\mathbf{K}(\operatorname{Inj} \mathcal{A})$ an isomorphism

$$
\operatorname{Hom}_{\mathbf{K}(\mathcal{A})}\left(X \otimes_{k} i k, Y\right) \longrightarrow \operatorname{Hom}_{\mathbf{K}(\mathcal{A})}(X, Y) .
$$

This follows from Lemma 2.1, in addition using the formula (8.1).

(2) Fix an object $X$ in $\mathbf{K}(\operatorname{Inj} \mathcal{A})$. The map $X \cong X \otimes_{k} i k \rightarrow X \otimes_{k} t k$ induces for all $Y$ in $\mathbf{S}(\mathcal{A})$ an isomorphism

$$
\operatorname{Hom}_{\mathbf{K}(\mathcal{A})}\left(X \otimes_{k} t k, Y\right) \longrightarrow \operatorname{Hom}_{\mathbf{K}(\mathcal{A})}(X, Y)
$$

To see this, consider the exact triangle

$$
X \otimes_{k} p k \longrightarrow X \otimes_{k} i k \longrightarrow X \otimes_{k} t k \longrightarrow \Sigma\left(X \otimes_{k} p k\right) .
$$

Now use that

$$
\operatorname{Hom}_{\mathbf{K}(\mathcal{A})}\left(X \otimes_{k} p k, Y\right) \cong \operatorname{Hom}_{\mathbf{K}(\mathcal{A})}\left(p k, \operatorname{Hom}_{k}(X, Y)\right)=0
$$

since $\operatorname{Hom}_{k}(X, Y)$ is acyclic.

(3) We apply Proposition 7.13. First observe that every object in $\mathcal{A}$ is Gorenstein injective. The functor

$$
\mathcal{A} \stackrel{\text { can }}{\longrightarrow} \mathbf{K}(\mathcal{A}) \stackrel{-\otimes t k}{\longrightarrow} \mathbf{S}(\mathcal{A})
$$

is naturally isomorphic to the stabilization functor $S: \mathcal{A} \rightarrow \mathbf{S}(\mathcal{A})$. This follows from the fact that $A \otimes_{k} i k$ is an injective resolution in $\mathcal{A}$ for each object $A$. Thus,

$$
S A \cong\left(A \otimes_{k} i k\right) \otimes_{k} t k \cong A \otimes_{k} t k .
$$

In Proposition 7.13, it is shown that $S$ induces an equivalence $\underline{\mathcal{A}} \rightarrow \mathbf{S}(\mathcal{A})$, with quasi-inverse $Z^{0}$.

Remark 8.3. The unit of the product in $\mathbf{K}(\operatorname{Inj} \mathcal{A})$ is $i k$, and its graded endomorphism ring is the cohomology ring $H^{*}(\Lambda, k)$. The unit of the product in $\mathbf{S}(\mathcal{A})$ is $t k$, and its graded endomorphism ring is the Tate cohomology ring $\widehat{H}^{*}(\Lambda, k)$.

\section{Appendix A. The DG category of noetherian objects}

Let $\mathcal{A}$ be a locally noetherian Grothendieck category. We give an alternative description of the homotopy category $\mathbf{K}(\operatorname{Inj} \mathcal{A})$ as the derived category of some DG category. Here, we follow closely Keller's exposition in [Kel94].

Let $\mathcal{C}$ be a small DG category. We recall the definition of the derived category $\mathbf{D}_{\mathrm{dg}}(\mathcal{C})$ of $\mathcal{C}$. The category $\mathbf{C}_{\mathrm{dg}}(\mathcal{C})$ of cochain complexes by definition has all DG $\mathcal{C}$-modules as objects. A map in $\mathbf{C}_{\mathrm{dg}}(\mathcal{C})$ is a map of $\mathrm{DG} \mathcal{C}$-modules which is homogeneous of degree zero and commutes with the differential. The homotopy category $\mathbf{K}_{\mathrm{dg}}(\mathcal{C})$ is obtained from $\mathbf{C}_{\mathrm{dg}}(\mathcal{C})$ by identifying homotopy 


\section{The STABLE DERIVED CATEGORY OF A NOETHERIAN SCHEME}

equivalent maps, where $f, g: X \rightarrow Y$ are homotopy equivalent if there exists a map $s: X \rightarrow Y$ of graded modules which is homogeneous of degree -1 and satisfies

$$
(f-g)^{n}=s^{n+1} \circ d+d \circ s^{n} \quad \text { for all } n \in \mathbb{Z} .
$$

Finally, the derived category of $\mathcal{C}$ is obtained from $\mathbf{K}_{\mathrm{dg}}(\mathcal{C})$ as the localization

$$
\mathbf{D}_{\mathrm{dg}}(\mathcal{C})=\mathbf{K}_{\mathrm{dg}}(\mathcal{C})\left[Q^{-1}\right]
$$

with respect to the class $Q$ of all maps $f$ which induce an isomorphism $H^{*} f$.

Given two cochain complexes $X$ and $Y$ in $\mathcal{A}$, we define the cochain complex $\mathcal{H}_{\mathcal{A}}(X, Y)$. The $n$th component is

$$
\prod_{p \in \mathbb{Z}} \operatorname{Hom}_{\mathcal{A}}\left(X^{p}, Y^{n+p}\right)
$$

and the differential is given by

$$
d\left(f^{p}\right)=d \circ f^{p}-(-1)^{n} f^{p+1} \circ d .
$$

Now fix a class $\mathcal{C}$ of objects in $\mathcal{A}$. We obtain a DG category $\overline{\mathcal{C}}$ by taking as objects for each $A$ in $\mathcal{C}$ an injective resolution $\bar{A}$, and as maps

$$
\operatorname{Hom}_{\overline{\mathcal{C}}}(\bar{A}, \bar{B})=\mathcal{H o m}_{\mathcal{A}}(\bar{A}, \bar{B}) .
$$

Proposition A.1. Let $\mathcal{A}$ be a locally noetherian Grothendieck category, and let $\mathcal{C}$ be a class of noetherian objects which generate $\mathbf{D}^{\mathrm{b}}($ noeth $\mathcal{A})$; that is, there is no proper thick subcategory containing $\mathcal{C}$. Then the functor

$$
\mathbf{K}(\operatorname{Inj} \mathcal{A}) \longrightarrow \mathbf{D}_{\mathrm{dg}}(\overline{\mathcal{C}}),\left.\quad X \mapsto \mathcal{H}_{\mathcal{A}}(-, X)\right|_{\overline{\mathcal{C}}}
$$

is an equivalence of triangulated categories.

Proof. The functor is exact. To see that it preserves coproducts, fix an object $A$ in $\mathcal{C}$ and a family of objects $X_{i}$ in $\mathbf{K}(\operatorname{Inj} \mathcal{A})$. Then for every $n \in \mathbb{Z}$ we have

$$
\begin{aligned}
H^{n} \coprod_{i} \mathcal{H o m}_{\mathcal{A}}\left(\bar{A}, X_{i}\right) & \cong \coprod_{i} H^{n} \mathcal{H o m}_{\mathcal{A}}\left(\bar{A}, X_{i}\right) \cong \coprod_{i} \operatorname{Hom}_{\mathbf{K}(\operatorname{Inj} \mathcal{A})}\left(\Sigma^{-n} \bar{A}, X_{i}\right) \\
& \cong \operatorname{Hom}_{\mathbf{K}(\operatorname{Inj} \mathcal{A})}\left(\Sigma^{-n} \bar{A}, \coprod_{i} X_{i}\right) \cong H^{n} \mathcal{H o m}_{\mathcal{A}}\left(\bar{A}, \coprod_{i} X_{i}\right)
\end{aligned}
$$

since $\bar{A}$ is compact in $\mathbf{K}(\operatorname{Inj} \mathcal{A})$ by Lemma 2.1. Thus, the canonical map

$$
\left.\left.\coprod_{i} \mathcal{H o m}_{\mathcal{A}}\left(-, X_{i}\right)\right|_{\overline{\mathcal{C}}} \longrightarrow \mathcal{H} m_{\mathcal{A}}\left(-, \coprod_{i} X_{i}\right)\right|_{\overline{\mathcal{C}}}
$$

is an isomorphism. Furthermore, the functor induces for objects $A$ and $B$ in $\mathcal{C}$ bijections

$$
\operatorname{Hom}_{\mathbf{K}(\operatorname{Inj} \mathcal{A})}\left(\bar{A}, \Sigma^{n} \bar{B}\right) \cong H^{n} \mathcal{H o m}_{\mathcal{A}}(\bar{A}, \bar{B}) \cong H^{n} \operatorname{Hom}_{\overline{\mathcal{C}}}(\bar{A}, \bar{B}) \cong \operatorname{Hom}_{\mathbf{D}_{\mathrm{dg}}(\overline{\mathcal{C}})}\left(\bar{A}^{\wedge}, \Sigma^{n} \bar{B}^{\wedge}\right),
$$

where $\bar{A}^{\wedge}$ denotes the free module $\operatorname{Hom}_{\overline{\mathcal{C}}}(-, \bar{A})$. Using infinite dévissage, we conclude that the functor is fully faithful since $\mathcal{C}$ generates $\mathbf{K}(\operatorname{Inj} \mathcal{A})$. The functor is, up to isomorphism, surjective on objects since the image contains the free $\overline{\mathcal{C}}$-modules which generate $\mathbf{D}_{\mathrm{dg}}(\overline{\mathcal{C}})$.

Corollary A.2. Viewing noeth $\mathcal{A}$ as a $D G$ category, we have an equivalence

$$
\mathbf{K}(\operatorname{Inj} \mathcal{A}) \stackrel{\sim}{\longrightarrow} \mathbf{D}_{\mathrm{dg}}(\text { noeth } \mathcal{A}) .
$$

We remark that the proof of Proposition A.1 works for any homotopy category. To be precise, let $\mathcal{X}$ be an additive category with arbitrary coproducts and let $\mathcal{C}$ be a set of objects in $\mathbf{K}(\mathcal{X})$ which 


\section{H. KRAUSE}

are compact (when viewed as objects in the localizing subcategory generated by $\mathcal{C}$ ). Define $\overline{\mathcal{C}}$ as before by

$$
\operatorname{Hom}_{\overline{\mathcal{C}}}(A, B)=\mathcal{H o m}_{\mathcal{X}}(A, B)
$$

for $A$ and $B$ in $\mathcal{C}$. Then the functor

$$
\mathbf{K}(\mathcal{X}) \longrightarrow \mathbf{D}_{\mathrm{dg}}(\overline{\mathcal{C}}),\left.\quad X \mapsto \mathcal{H} m_{\mathcal{X}}(-, X)\right|_{\overline{\mathcal{C}}},
$$

induces an equivalence between the localizing subcategory which is generated by $\mathcal{C}$, and $\mathbf{D}_{\operatorname{dg}}(\overline{\mathcal{C}})$.

\section{Appendix B. Homotopically minimal complexes}

A complex $X$ in some additive category is called homotopically minimal, if every map $\phi: X \rightarrow X$ of complexes is an isomorphism provided there is a map $\psi: X \rightarrow X$ such that $\phi \circ \psi$ and $\psi \circ \phi$ are chain homotopic to the identity map $\operatorname{id}_{X}$. In this appendix, we show that each complex with injective components admits a decomposition $X=X^{\prime} \amalg X^{\prime \prime}$ such that $X^{\prime}$ is homotopically minimal and $X^{\prime \prime}$ is null homotopic.

Let $\mathcal{A}$ be an abelian category, and suppose that $\mathcal{A}$ admits injective envelopes. Given a complex $X$ in $\mathcal{A}$ with injective components, we construct for each $n \in \mathbb{Z}$ a new complex $X(n)$ as follows. Let $U^{n} \subseteq X^{n}$ be the injective envelope of $Z^{n} X$. We get a decomposition $X^{n}=U^{n} \amalg V^{n}$. Let $V^{n+1}$ be the image of $V^{n}$ under the differential $X^{n} \rightarrow X^{n+1}$, and let $V^{p}=0$ otherwise. This gives a complex $V$ which is null homotopic. The canonical map $\iota: V \rightarrow X$ is a split monomorphism in each degree. Thus, $\iota$ has a left inverse and we obtain a decomposition $X=U \amalg V$. We put $X(n)=V$.

Lemma B.1. Let $\mathcal{A}$ be an abelian category, and suppose that $\mathcal{A}$ admits injective envelopes. Then the following are equivalent for a complex $X$ in $\mathcal{A}$ with injective components.

(1) The complex $X$ is homotopically minimal.

(2) The complex $X$ has no non-zero direct factor which is null homotopic.

(3) The canonical map $Z^{n} X \rightarrow X^{n}$ is an injective envelope for all $n \in \mathbb{Z}$.

Proof. (1) $\Rightarrow(2)$ Let $X=X^{\prime} \amalg X^{\prime \prime}$ and suppose that $X^{\prime}$ is null-homotopic. The idempotent map $\varepsilon: X \rightarrow X$ with $\operatorname{Ker} \varepsilon=X^{\prime}=$ Coker $\varepsilon$ induces an isomorphism in the homotopy category. Thus (1) implies $X^{\prime}=0$.

$(2) \Rightarrow(3)$ Fix $n \in \mathbb{Z}$. Then we have a decomposition $X=X(n) \amalg U$ such that $X(n)$ is null homotopic. Our assumption implies $X(n)=0$, and we conclude that the map $Z^{n} X \rightarrow X^{n}$ is an injective envelope.

(3) $\Rightarrow(1)$ Let $\phi: X \rightarrow X$ be a map with inverse $\psi$ such that $\psi \circ \phi$ and $\phi \circ \psi$ are chain homotopic to the identity $\operatorname{id}_{X}$. Thus, we have a family of maps $\rho^{n}: X^{n} \rightarrow X^{n-1}$ such that

$$
\operatorname{id}_{X^{n}}=(\psi \circ \phi)^{n}+\delta^{n-1} \circ \rho^{n}+\rho^{n+1} \circ \delta^{n} .
$$

We claim that $\operatorname{Ker} \phi=0$. In fact, we show that $K=\operatorname{Ker}(\psi \circ \phi)=0$. Let $L^{n}=K^{n} \cap Z^{n} X$. Then $\rho^{n}$ identifies $L^{n}$ with $\rho^{n}\left(L^{n}\right)$, and $\rho^{n}\left(L^{n}\right) \cap Z^{n-1} X=0$, since $\left(\delta^{n-1} \circ \rho^{n}\right) L^{n}=L^{n}$. The assumption on $Z^{n-1} X$ implies $L^{n}=0$. The same assumption on $Z^{n} X$ implies $K^{n}=0$. Let $C=$ Coker $\phi$. The sequence $0 \rightarrow X \stackrel{\phi}{\rightarrow} X \rightarrow C \rightarrow 0$ is split exact in each degree because $X$ has injective components. It follows that the sequence is split exact in the category of complexes, because $C$ is null homotopic by our assumption on $\phi$. Let $\phi^{\prime}: X \rightarrow X$ be a left inverse of $\phi$. Then Ker $\phi^{\prime} \cong C$. On the other hand, $\phi^{\prime}$ is invertible in the homotopy category of complexes and therefore $\operatorname{Ker} \phi^{\prime}=0$ by the first part of this proof. Thus $\phi$ is an isomorphism.

Proposition B.2. Let $\mathcal{A}$ be an abelian category, and suppose that $\mathcal{A}$ admits injective envelopes. Then every complex $X$ in $\mathcal{A}$ with injective components has a decomposition $X=X^{\prime} \amalg X^{\prime \prime}$ such 


\section{THE STABLE DERIVED CATEGORY OF A NOETHERIAN SCHEME}

that $X^{\prime}$ is homotopically minimal and $X^{\prime \prime}$ is null homotopic. Given a second decomposition $X=$ $Y^{\prime} \amalg Y^{\prime \prime}$ such that $Y^{\prime}$ is homotopically minimal and $Y^{\prime \prime}$ is null homotopic, then the canonical map $X^{\prime} \longmapsto X \rightarrow Y^{\prime}$ is an isomorphism.

Proof. Take $X^{\prime \prime}=\coprod_{n \in \mathbb{Z}} X(n)$. This complex is null homotopic and the canonical map $\iota: \coprod_{n \in \mathbb{Z}} X(n)$ $\rightarrow X$ is a split monomorphism in each degree. Thus, $\iota$ has a left inverse and we obtain a decomposition $X=X^{\prime} \amalg X^{\prime \prime}$. The construction of each $X(n)$ shows that the inclusion $Z^{n}\left(X^{\prime}\right) \rightarrow\left(X^{\prime}\right)^{n}$ is an injective envelope. Thus, $X^{\prime}$ is homotopically minimal, by Lemma B.1.

Now let $X=Y^{\prime} \amalg Y^{\prime \prime}$ be a second decomposition such that $Y^{\prime}$ is homotopically minimal and $Y^{\prime \prime}$ is null homotopic. The canonical map $\phi: X^{\prime} \longmapsto X \rightarrow Y^{\prime}$ induces an isomorphism in the homotopy category, since $X^{\prime \prime}$ and $Y^{\prime \prime}$ are null homotopic. Thus, $\phi$ is an isomorphism of complexes, since $X^{\prime}$ and $Y^{\prime}$ are homotopically minimal. This completes the proof.

\section{ACKNowledgements}

It is a pleasure to thank a number of colleagues for their interest in the topic of this paper. Øyvind Solberg helped to prove the crucial Proposition 2.3. Peter Jørgensen pointed out the relevance of the stable derived category in algebraic geometry. Amnon Neeman communicated an alternative proof of Corollary 4.4 in the algebraic geometric context. Ragnar Buchweitz explained the connection with his earlier unpublished work. Apostolos Beligiannis provided numerous comments on a preliminary version of this manuscript. Finally, I wish to thank the anonymous referee for many helpful suggestions.

\section{REFERENCES}

AB69 M. Auslander and M. Bridger, Stable module theory, Mem. Amer. Math. Soc., vol. 94 (American Mathematical Society, Providence, RI, 1969).

AB89 M. Auslander and R.-O. Buchweitz, Maximal Cohen-Macaulay approximations, Mém. Soc. Math. Fr. (N.S.) 38 (1989), 5-37.

AM02 L. L. Avramov and A. Martsinkovsky, Absolute, relative, and Tate cohomology of modules of finite Gorenstein dimension, Proc. London Math. Soc. (3) 85 (2002), 393-440.

BBD82 A. A. Beilinson, J. Bernstein and P. Deligne, Faisceaux pervers, Astérisque 100 (1982).

BEH87 R.-O. Buchweitz, D. Eisenbud and J. Herzog, Cohen-Macaulay modules on quadrics, in Singularities, representation of algebras, and vector bundles, Lecture Notes in Mathematics, vol. 1273 (Springer, Berlin, 1987), 58-116.

Bel00 A. Beligiannis, The homological theory of contravariantly finite subcategories: Auslander-Buchweitz contexts, Gorenstein categories and (co-)stabilization, Comm. Algebra 28 (2000), 4547-4596.

BG04 D. J. Benson and J. P. C. Greenlees, Localization and duality in topology and modular representation theory, Preprint (2004).

BGG78 J. N. Bernstein, I. M. Gelfand and S. I. Gelfand, Algebraic bundles over $\mathbb{P}^{n}$ and problems of linear algebra, Funktsional. Anal. i Prilozhen. 12 (1978), 66-67.

BGS96 A. Beilinson, V. Ginzburg and W. Soergel, Koszul duality patterns in representation theory, J. Amer. Math. Soc. 9 (1996), 473-527.

BN93 M. Bökstedt and A. Neeman, Homotopy limits in triangulated categories, Compositio Math. 86 (1993), 209-234.

BR02 A. Beligiannis and I. Reiten, Homological and homotopical aspects of torsion theories, Preprint (2002), 200 pp.

BV03 A. Bondal and M. van den Bergh, Generators and representability of functors in commutative and noncommutative geometry, Mosc. Math. J. 3 (2003), 1-36. 


\section{THE STABLE DERIVED CATEGORY OF A NOETHERIAN SCHEME}

Buc87 R.-O. Buchweitz, Maximal Cohen-Macaulay modules and Tate-cohomology over Gorenstein rings, Unpublished manuscript (1987), 155 pp.

EJ95 E. E. Enochs and O. M. G. Jenda, Gorenstein injective and projective modules, Math. Z. 220 (1995), 611-633.

EJ00 E. E. Enochs and O. M. G. Jenda, Relative homological algebra (de Gruyter, Berlin, 2000).

Gabb62 P. Gabriel, Des catégories abéliennes, Bull. Soc. Math. France 90 (1962), 323-448.

Har66 R. Hartshorne, Residues and duality, Lecture Notes in Mathematics, vol. 20 (Springer, Berlin, 1966).

Hov02 M. Hovey, Cotorsion pairs, model category structures, and representation theory, Math. Z. 241 (2002), 553-592.

HPS97 M. Hovey, J. H. Palmieri and N. P. Strickland, Axiomatic stable homotopy theory, Mem. Amer. Math. Soc., vol. 610 (American Mathematical Society, Providence, RI, 1997).

Jor01 P. Jørgensen, Spectra of modules, J. Algebra 244 (2001), 744-784.

Jor03 P. Jørgensen, A non-commutative BGG-correspondence, Preprint (2003).

Jor05 P. Jørgensen, The homotopy category of complexes of projective modules, Adv. Math. 193 (2005), 223-232.

Kel94 B. Keller, Deriving DG categories, Ann. Sci. École Norm. Sup. (4) 27 (1994), 63-102.

Kra02 H. Krause, A Brown representability theorem via coherent functors, Topology 41 (2002), 853-861.

KV87 B. Keller and D. Vossieck, Sous les catégories dérivées, C. R. Acad. Sci. Paris Sér I Math. 305 (1987), 225-228.

Mis94 G. Mislin, Tate cohomology for arbitrary groups via satellites, Topology Appl. 56 (1994), 293-300.

Nee92 A. Neeman, The connection between the $K$-theory localization theorem of Thomason, Trobaugh and Yao and the smashing subcategories of Bousfield and Ravenel, Ann. Sci. École Norm. Sup. (4) 25 (1992), 547-566.

Nee96 A. Neeman, The Grothendieck duality theorem via Bousfield's techniques and Brown representability, J. Amer. Math. Soc. 9 (1996), 205-236.

Nee01 A. Neeman, Triangulated categories, Annals of Mathematics Studies, vol. 148 (Princeton University Press, Princeton, NJ, 2001).

Nuc98 B. E. A. Nucinkis, Complete cohomology for arbitrary rings using injectives, J. Pure Appl. Algebra 131 (1998), 297-318.

Orl03 D. Orlov, Triangulated categories of singularities and D-branes in Landau-Ginzburg models, Proc. Steklov Inst. Math. 3 (246) (2004), 227-248.

Ren69 R. Rentschler, Sur les modules $M$ tels que $\operatorname{Hom}(M,-)$ commute avec les sommes directes, C. R. Acad. Sci. Paris Ser. A 268 (1969), 930-933.

Ric89 J. Rickard, Derived categories and stable equivalence, J. Pure Appl. Algebra 61 (1989), 303-317.

Spa88 N. Spaltenstein, Resolutions of unbounded complexes, Compositio Math. 65 (1988), 121-154.

Ver96 J. L. Verdier, Des catégories dérivées des catégories abéliennes, Astérisque 239 (1996).

Henning Krause hkrause@math.uni-paderborn.de

Institut für Mathematik, Universität Paderborn, 33095 Paderborn, Germany 